
\title{
BResarch Soute \\ Optimization Method for an Information Interaction Interface from Error-Cognition to Information Feature Mapping
}

Xiaoli Wu ( $\nabla$ wuxlhhu@163.com)

Nanjing University of Science \& Technology

Sabrina Caldwell

Australian national University

\section{Original Article}

Keywords: Human computer interaction, Human Error, Visual Information Features, Error-cognition

Posted Date: May 29th, 2020

DOI: https://doi.org/10.21203/rs.3.rs-30232/v1

License: (c) (i) This work is licensed under a Creative Commons Attribution 4.0 International License.

Read Full License 


\title{
Optimization Method for an Information Interaction Interface from
}

\section{Error-Cognition to Information Feature Mapping}

\author{
Xiaoli Wu*a,b,c,d, Sabrina Caldwellb \\ ${ }^{a}$ School of Design Art \& Medial, Nanjing University of Science \& Technology, 210094 Nanjing, China \\ ${ }^{b}$ Research School of Computer Science, The Australian National University, ACT, Australia \\ ${ }^{c}$ College of Mechanical and Electrical Engineering, Hohai University, Changzhou, China \\ ${ }^{d}$ Lab of Human Factors and Information System Interaction \& Design, Hohai University, Changzhou, China
}

\begin{abstract}
Background- With the rapid development of digital and intelligent information systems, display of visual information in interfaces has become an important challenge in the field of human-computer interaction. Objective- We propose a method for the optimization of information interaction interfaces from error-cognition through the mapping of information characteristics. Method- A mapping method of matrix description is adopted to analyze the association properties between error-cognition sets and design information sets. Results- Based on the mapping relationship between the domains of error-cognition and design information, a cross-correlational analysis is carried out between error-cognition and design information. Conclusion- We obtain the relationship matrix between the error-cognition of correlation between design information and the degree of importance among design information. Application- Taking the task interface of a warfare navigation display as an example, error factors and the features of design information are extracted. Based on the results, we also propose an optimization design scheme for the monitoring task interface.
\end{abstract}

Keywords: Information interaction interface, error-cognition, information feature mapping, visual information display, optimization

\section{Description}

We study the mapping between error, cognition and design. The transition from the mapping of error and cognitive domains to the mapping of design domain enables the process of optimizing interface information. The design solution process can be developed by importing error factors and combining them with visual cognition.

\section{Introduction}

\subsection{Information Interaction Interface Literature Review}

The human-computer interaction industry has long been exploring reasonable and feasible design methods to improve the information presentation problem faced by current digital, intelligent visual information interfaces. Especially in the field of complex information systems, researchers both at home and abroad have devoted time and effort to seek reasonable methods for information display through research on information coding and layouts. For instance, in the Human Measures and Performance Project (HMPP), NASA (1996) specifically studied the problems of color security and availability of the design of various complex graph display interfaces in the aviation field. Michelle and Wickens (2001) carried out experiments to investigate how to best exhibit relevant electronic information on battlefield maps. Montgomery and

*Corresponding author. Email:wuxlhhu@163.com 
Sorkin (1996) performed an experimental study on the effect of luminance on an observer's degree of reliability in identifying information in a visual display. Tullis (1981) and Schum (1991) investigated the efficiency of identifying digital and graphical information coding. Patrick Monnier (2003) applied the experimental paradigm of visual delay in search tasks to study the relationship between colors and locations. Parsons et al. (2014) summarized ten attributes of interactive visual displays (including importance, relations and adjustability of interactions). Li Liangming, in 1984, at an early stage of the aviation industry in our country, analyzed and investigated the usage of circular scale instruments in different types of domestic airplane pilot seats to propose suggestions to improve their instrumental scales, pointers, digital display, benchmark of flight attitude, instrumental size and layout. Liu Baoshan and Ding Yaping (1992) studied the recognition effects of the relative locations of display interface in instrument panels of fighter planes and helicopters. Heather et al. (2012) performed research on the information representation of auto-battle recognition system. Lei Zhou, Jing Li, Yafeng Niu, Xiaoli Wu, Tao Jin, and Chengqi Xue et al. (2013 2015) performed physiological research on the information representation and interface design of human-machine interfaces of complex systems. All these works of research indicate the importance of information presentation in the process of monitoring task execution. They conclude that icons, symbols and colors are critical styles of expression for information presentation.

\subsection{Error Factors Literature Review}

With the rapid development of computer graphics and user interface technologies, the study of human errors has also been applied to the field of interface design to improve interface effectiveness. Nielsen (1994) and Shryane (1998) proposed methods for reducing the error rates to improve interface efficiency. Li Leshan (2004) proposed a system for the error classification of human-computer interfaces. He proposed that inattention and over-attention can both be regarded as key ideas which need to be taken into consideration in the research on human errors. Hassnert and Allwood (2002) concluded that it is not possible to have a uniform standard for error classification. They stated that the corresponding types of human errors should be identified through experimental methods. They conducted inductive classification towards user errors by means of user interface tests of the software. Krokos and Baker proposed error classification methods for interface cognition. Maxion and Reeder (2005) investigated the use of the external subgoal support method to enhance the reliability of user interfaces by reducing errors. Javier (2013) used a driver behavior questionnaire to analyze the reasons caused by attention errors (driving errors, traffic violations and excessive behaviors) and found the key factors causing operational errors. Shappell (2007) analyzed aircraft accidents data in 2013 and also identified attention, comprehension and other related factors corresponding to skills and decision-making. Anokhin (2009) analyzed the ergonomics of control panel design of main control room in nuclear power station, concluding that the most typical design error is incompatibility between irregular layout and equipment; Shen Zupei (2009) established the theory and method of the human error cognition model; Wang Pei and Pei-luen Rau (2011) established an operator behavior model from the perspective of human factors engineering and decision-making behavior; Li Pengcheng (2011) established an evaluation model and a reliability evaluation method of nuclear power plant digital control system based on operator situation cognition; Wang Meng and Zhang Yijing (2014) analyzed the reason for operation mistakes in aerospace and provided a reference for improving astronaut operation performance.

This paper proposes a framework called the "Error-Cognition-Design", based on our study of the association between error factors, visual cognition and design information. This framework offers a technique that can map the mechanism of error-cognition into the design factors of information interfaces, to perform optimization for the design of complex information interfaces.

\section{The Optimized Method of Information Interaction Interface}


In the research field of design methodology, the design process is regarded as a system that transforms a model from a concept-function to a structural mapping. This has been studied in depth since the end of the last century, and has been applied to the fields of machinery and product design. The direct mapping from a function to a structure was proposed by Pahl and Beitz in 1992 as a typical representation in the field. In 2000, Suh proposed an alternate reciprocating mapping in terms of function-structure mapping and structure-subfunction mapping. Based on Pahl's work, in 1996, Gero proposed a multi-level mixed mapping method which introduced a behavioral domain between the functional and structural domains. In the above-mentioned studies, the process of design solution is formed by mapping methods such as matrix description and genetic algorithms. This process opens up an effective method for the design of mechanical structures that can be widely applied to the innovative design of products. In the human-computer interaction field, for the optimization of design methods for complex information interfaces, we must discuss the feasibility of the designs from the perspective of error-cognition to design mapping. It needs to be seen whether this is able to open up a solution process for interface design while learning from error factors.

In this paper, we study the possible mapping between error, cognition and design. The transition from the mapping of error and cognitive domains to the mapping of a design domain enables the process of optimizing interface efficiency. The design solution process of a complex information interface can be developed by importing error factors and combining them with the visual cognition behavior of the information interface.

The optimization method of an information interaction interface from error-cognition to information feature mapping could provide a reliable analytical solution. This procedure is summarized as follows (Figure 1) :

1 ) given an error-cognition domain, we can determine the degree of relative importance of the error-cognition from the cross correlation between domain factors, and then the association properties of error-cognition can be solved;

2 ) we can establish a design information domain from analysizing design factors of information features. Using the same method of error-cognition, the association properties of the design information feature can be solved;

3) we can establish the design solution process from error-cognition to information feature mapping. We also need to determine the objective constraints of design information features;

4) in the case of task interface design of typical display systems, we can extract error factors based on the monitoring interface tasks and then obtain the solution process from error-cognition to information feature mapping from extracted error factors. 


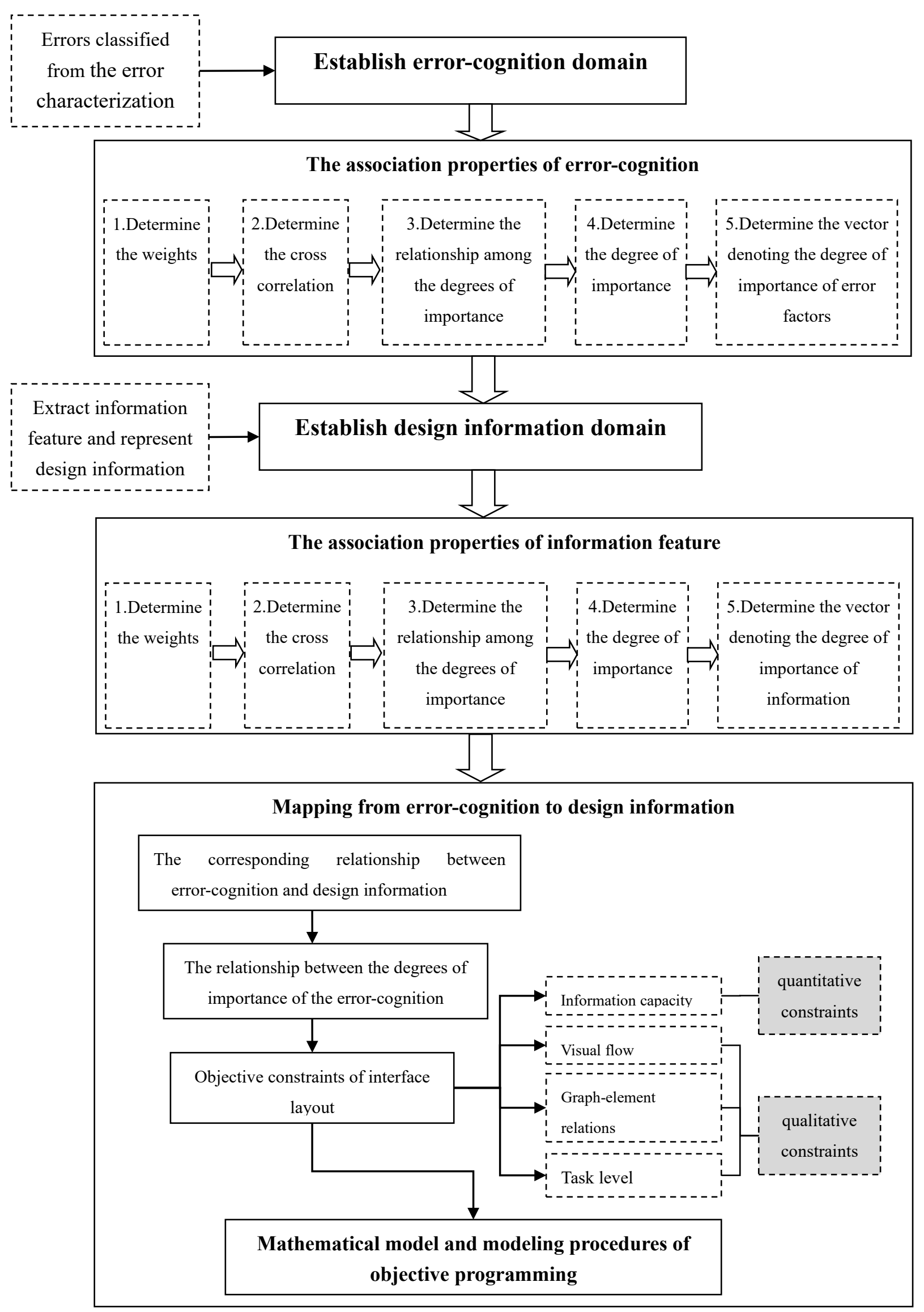

Figure 1. Procedure of the optimization method 


\section{Error-cognition factors and design information factors}

\subsection{Error factors of information interaction interface}

In information interaction interfaces, for example an aviation information display, there are several possible tasks to be executed, such as monitoring status data, querying task information, monitoring threat and security state information, and so on. Display interfaces of complex information system display navigation, situation pictures, status data and other information. The monitoring task is likely to be performed: plan creating, state monitoring, burst scheduling, and so on. We can classify the monitoring interfacial task either by abrupt events and common tasks, or by the order in which tasks are performed. Thus, as shown in Table 1, we listed the monitoring interface tasks and corresponding error factors to extract the error characterization of a monitoring interface of a complex system.

Table 1 Characterization of error factor of monitoring interface task (Xiaoli Wu, 2016)

\begin{tabular}{|c|c|c|c|c|}
\hline $\begin{array}{c}\text { Tasks of monitoring } \\
\text { interface A }\end{array}$ & $\begin{array}{l}\text { Display format of } \\
\text { information B }\end{array}$ & $\begin{array}{c}\text { Cognitive behavior } \\
\mathrm{C}\end{array}$ & Error factor D & Representation of error E \\
\hline A1 monitor/discover & B1 dynamic display & C1 search & D1 ignorance & E1 ambiguity states \\
\hline A2 inquire state & B2 static display & C2 recognize & D2 omission & E2 visual limitation \\
\hline A3 plan response & $\mathrm{B} 3$ navigation & C3 identify & D3 miss & E3 visual bluntness \\
\hline \multirow[t]{19}{*}{ A4 execute response } & B4 status data & C4 judge \& select & D4 misreading & E4 visual illusion \\
\hline & B5 information icon & C5 decision-making & D5 misjudgment & E5 attentional load \\
\hline & B6 alarm reminder & & D6 misunderstanding & E6 visual disturbance \\
\hline & & & D7 haven't seen & E7 overattention \\
\hline & & & D8 confusion & E8 attention shift and distraction \\
\hline & & & D9 cannot remember & E9 too nervous to do anything \\
\hline & & & D10 input error & E10 cognitive bias \\
\hline & & & D11 misregistration & E11 unreasonable match \\
\hline & & & D12 cannot see clearly & E12 weak visibility \\
\hline & & & D13 hard to distinguish & E13 thinking load \\
\hline & & & D14 match incorrectly & E14 forget \\
\hline & & & D15 cannot find & E15 inaccurate recall \\
\hline & & & D16 delay & E16 lack of memory aids \\
\hline & & & D17 inadequate & E17 intentionality decrease \\
\hline & & & D18 irrelevant & E18 false memory \\
\hline & & & D19 react too early & E19 unconsciousness \\
\hline & & & D20 no reaction & E20 omission caused by inattention \\
\hline & & & D21 select incorrectly & E21 time pressure \\
\hline & & & D22 slip & \\
\hline
\end{tabular}

\subsection{Information features of information interaction interface}

As the analysis object of the design information characteristic, the monitoring task interface of some kind of navigation war is divided into four parts based on different tasks: (i) radar situation interface, (ii) weapon mounting interface, (iii) multi-sensor interface and (iv) flight data display interface, including the navigation, situation charts, state data, alarm reminder and other information display. There are four processes in monitoring tasks which may be performed such as monitoring/detection, state query, response planning and response execution. Table 2 shows the information content displayed in the different monitoring task interfaces, which will be regarded as the content of main information in searching, reading, recognition, judging selection and decision making. The radar situation interface mainly displays the information of the aircraft radar area including the 
appearance of the target, the database calling, the different aircraft symbols in the range, the attacked target display, the driving route and other information. The weapon mounting interface mainly displays the selection of weapons, display, launch selection, the current state of selection and the weapon programming, etc; The multi-sensor display interface mainly displays radar setting and selection, satellite map information, radar proportional rendering and other information. The flight data display interface mainly displays the machmeter, forecast speed, attack angle, height, horizontal meter calibration, fuel and other indicators.

Table 2 monitoring task interface of typical navigation war display system

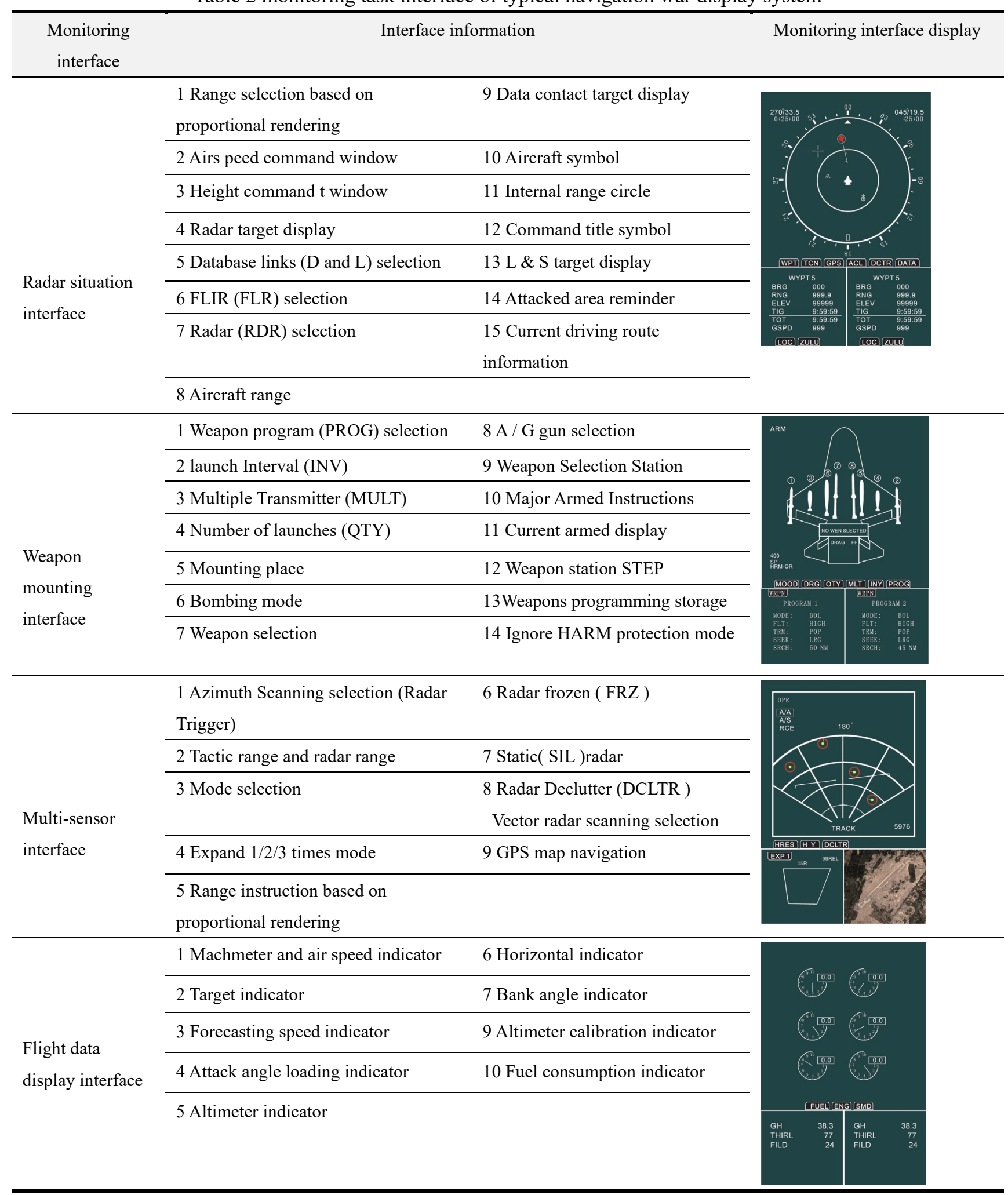




\section{The Solution Process from Error-cognition to Information Feature Mapping}

\subsection{Analysis of the Association Properties of Error-Cognition}

First, we need to analyze the association properties of the error-cognition domain. Let $R_{\mathrm{E} 0}=\left\{r_{e 1}, r_{e 2}, \ldots\right.$, $\left.r_{e m 1}\right\}$ be the screened error-cognition set, where $r_{e i}\left(i=1,2, \ldots, m_{1}\right)$ denotes the error factor. Let us assume that $r_{e i}, r_{e j} \in R_{E 0}$.

Since the corresponding processes of cognitive information are different, the same error factor will not have corresponding inclusion and crossing relations. For instance, take the case of misreading caused by visual restrictions or unreasonable matching of information. These two conditions do not have the properties of inclusiveness or intersection (Xiaoli et al., 2014). They belong to error factors from different perspectives, and can be explained as errors resulting from different internal reasons. Hence, the relations $r_{e i}$ and $r_{e j}$ are both considered to be independent of each other. Hence, if the error factors included in $r_{e i}$ are not related to the error factors included in $r_{e j}$, we can state that $r_{e i}$ and $r_{e j}$ are independent.

With respect to the existing correlation between error and cognition (Xiaoli et al., 2014 ), when further screening the factors in the error-cognition domain, selections must be made among error-cognition factors with mutual exclusion. The choice for the appropriate factors must be made based on the sequence of the degrees of importance of the factors.

After screening, the error-cognition set can be denoted as $R_{E}^{\prime}=\left\{r_{\mathrm{e} 1}, r_{\mathrm{e} 2}, \ldots, r_{\mathrm{e} p}\right\}$, where $r_{\mathrm{e} i}(i=1,2, \ldots, p)$ represents the error-cognition factor. For error-cognition factors with no correlation, mutual conflict or mutual collaboration, we refine them by sorting them based on their degrees of importance and deleting the unimportant ones.

In this paper, the method of Analytic Network Process (ANP) is employed to determine the degree of importance between error and cognition (Lee, 2000). This procedure of using ANP is summarized as follows:

Step 1: Without considering the correlation between error and cognition, the weights of error-cognition are determined. A numerical sequence of 1 to 9 is adopted for notation, and a normalization is conducted to obtain the error-cognition weight vector $w_{r}, w_{r}=\left(w_{1}, w_{2}, \ldots, w_{p}\right)^{T}$, where $\sum_{i=1}^{p} w_{i}=1$.

Step 2: Determine the cross correlation between the error and cognition. The matrix $\boldsymbol{E}$ is used to describe such cross correlation. 1-3-9 is employed to express the weak, intermediate and strong cooperative relations between error and cognition; (-1)-(-3)-(-9) is adopted to denote the weak, intermediate and strong conflict relations between error and cognition. 0 denotes the irrelevance of error-cognition, where $e_{i j}=e_{j i}, e_{i i}$ $=9$.

$$
\mathbf{E}=\left[\begin{array}{cccc}
e_{11} & e_{12} & \cdots & e_{1 p} \\
e_{21} & e_{22} & \cdots & e_{2 p} \\
\vdots & \vdots & \ddots & \vdots \\
e_{p 1} & e_{p 2} & \cdots & e_{p p}
\end{array}\right]
$$

The correlation information for each involved error-cognition factor can be calculated by analyzing the cross-correlation matrix $\boldsymbol{E}$. Take the error-cognition factor $r_{e i}$ as an example.

1) Positive correlation number $R_{p i}$ denotes the number of error-cognition factors positively related to $r_{e i}$.

2) Negative correlation number $R_{n i}$ denotes the number of error-cognition factors negatively related to $r_{e i}$

3) Degree of positive correlation $R_{s p i}$ denotes the total sum of the correlation degrees of error-cognition 
factors that are positively related to $r_{e i}$.

4) Degree of negative correlation $R_{s p i}$ denotes the total sum of the correlation degrees of error-cognition factors that are negatively related to $r_{e i}$.

5) Average degree of correlation $R_{a v i}^{\prime}$ denotes the average value of the correlation degrees of error-cognition factors that are related to $r_{e i} . R_{a v i}^{\prime}$ is represented by: $R_{a v i}^{\prime}=\frac{1}{p} \sum_{j=1}^{p} e_{i j}$.

The information above is fundamental to determining the degree of relative importance of the error-cognition. It is also used to select the values of error-cognition that have the same degree of importance. The method to identify values of error-cognition with identical degrees of importance is as follows:

1) First, find out the subset of error-cognition for $R_{n i}=0(i=1,2, \ldots, p)$. Then sort the values of the error-cognition $R_{s p i}$ in each set in a descending order and make selections;

2 ) If the result of the last step does not yield the desired values, increase the value of $R_{n i}$ by 1 . Find out the corresponding subset of error-cognition, and select the error-cognition factor which has a large value for $R_{p i}$ and a large value for $R_{a v i}^{\prime}$. The value of error-cognition should also have a negative correlation with $R_{a v i}^{\prime}$, indicating a low degree of importance of error-cognition.

3 ) If the result of the last step does not satisfy the requirements, then return to 2) and repeat till the requirements are satisfied.

Step 3: Determine the relationship among the degrees of importance of the error-cognition. The matrix $W_{\mathrm{r}}$ is used to describe this relationship among the degrees of importance of the error-cognition. Take $r_{e i}$ as an example. Based on the consideration that there is a correlation between $r_{e i}$ and error-cognition, the degrees of relative importance of the other error-cognition factors with respect to $r_{e i}$ are determined to obtain a matrix $\boldsymbol{W}_{i}$.

Here $r_{i j} \in[0,9]$; when $r_{i j} \neq 0, r_{j i}=1 / r_{i j}, r_{j i}=0$ when $r_{i j}=0$. The AHP analytical method is employed to obtain the vector of the degrees of relative importance of the other error-cognition values specific to $r_{e i}$. This is represented as $w_{i}=\left(w_{1 i}, w_{2 i}, \ldots, w_{i i}, \ldots, w_{p i}\right)^{T}$, where $\sum_{j=1}^{p} w_{j i}=1$. Using this, we can solve the matrix for the degrees of importance of error- cognition $\boldsymbol{W}_{r}$ :

$$
\begin{aligned}
& \mathbf{W}_{i}=\left[\begin{array}{cccccc}
r_{11} & r_{12} & \cdots & r_{1 i} & \cdots & r_{1 p} \\
r_{21} & r_{22} & \cdots & r_{2 i} & & r_{2 p} \\
\vdots & \vdots & \ddots & \vdots & & \vdots \\
r_{i 1} & r_{i 2} & \cdots & r_{i \ddot{i}} & \cdots & r_{i p} \\
\vdots & \vdots & & \vdots & \vdots & \\
r_{p 1} & r_{p 2} & \cdots & r_{p i} & \cdots & r_{p p}
\end{array}\right] \\
& \mathbf{W}_{r}=\left[\begin{array}{cccccc}
w_{11} & w_{12} & \cdots & w_{1 i} & \cdots & w_{1 p} \\
w_{21} & w_{22} & \cdots & w_{2 i} & & w_{2 p} \\
\vdots & \vdots & \ddots & \vdots & & \vdots \\
w_{i 1} & w_{i 2} & \cdots & w_{i i} & \cdots & w_{i p} \\
\vdots & \vdots & & \vdots & \ddots & \vdots \\
w_{p 1} & w_{p 2} & \cdots & w_{p i} & \cdots & w_{p p}
\end{array}\right]
\end{aligned}
$$

Step 4: Determine the degree of importance for the error-cognition. The vector $w_{e}^{\prime}$ is used to denote the degree of importance for the error-cognition as:

$$
w_{e}^{\prime}=W_{r} \times w_{r}=\left(w_{\mathrm{el}}, w_{\mathrm{e} 1}, \ldots, w_{e p}\right) \text {. }
$$

Step 5: Determine the final error-cognition. According to the sequence of the degree of importance of 
the error-cognition, unimportant factors of error-cognition are removed. When the difference in the degree of importance of the error-cognition is not significant, the decision method in (2) is employed to make decisions. Then calculate the final error-cognition represented by $R_{E}=\left\{r_{e 1}, r_{e 2}, \ldots, r_{e m}\right\}$; and the vector denoting the degree of importance of error factors as $w_{E}=\left(w_{1}, w_{1}, \ldots, w_{m}\right)^{T}$, which corresponds to the normalized error-cognition set.

\subsection{Analysis of the Association Properties of the Design Information Features}

Operators can divide the monitoring tasks (Task $i$ ) into four processes. They are 1) surveillance/discovery, 2) status inquiry, 3) response planning and 4) response execution. Each process executes different tasks such as information search, information recognition and reading, information identification, information selection and judgment. Each of these tasks is associated with the relevant design information steps from 1 to n: $\operatorname{Design}\left(i_{1}\right)$, Design $\left(i_{2},\right), \cdots, \operatorname{Design}\left(i_{n^{\prime}}\right)$. Let $C_{Q 0}=\left\{c_{q 1}, c_{q 2}, \ldots, c_{q n 1}\right\}$ be the error-cognition set, where $c_{q i}(i=$ $\left.1,2, \ldots, n_{1}\right)$ represents the error factor, then for each $c_{q i}$, we have $c_{q j} \in C_{Q 0}$.

An Interface for monitoring complex information systems displays numerous complicated information items. Such a display may sometimes not be systematic and may even contain a certain level of redundancy. Thus, it may become necessary to arrange, filter and analyze the designed information features. There are three kinds of relations which exist between $c_{q i}$ and $c_{q j}$. They are inclusion, intersection and independence. The design information set after screening is denoted as $C^{\prime}{ }_{\mathrm{Q}}=\left\{c_{q 1}, c_{q 2}, \ldots, c_{q p}\right\}$, where each element $c_{q i}(i=$ $1,2, \ldots, p)$ is an information feature. During the design process, after removing redundancies, these features may contain relations such as mutual exclusion, irrelevance, mutual conflict and mutual collaboration.

The ANP method is next adopted to determine the degree of importance of the features for information design. First, we determine the weight of the information features, as well as the mutual relations among them to obtain the mutual correlation matrix $\mathbf{Q}$ :

$$
\mathrm{Q}=\left[\begin{array}{cccc}
q_{11} & q_{12} & \cdots & q_{1 p} \\
q_{21} & q_{22} & \cdots & q_{2 p} \\
\vdots & \vdots & \ddots & \vdots \\
q_{p 1} & q_{p 2} & \cdots & q_{p p}
\end{array}\right]
$$

The correlation information of each relevant feature can be obtained by analyzing the mutual correlation matrix $\mathbf{Q}$. Identical to the method used in the error-cognition set, taking the information feature $c_{q i}$ as an example, the positive correlation number $C_{p i}$, the negative correlation number $C_{n i}$, the positive correlation degree $C_{s p i}$, the negative correlation degree $C_{s n i}$ and the average correlation degree $C_{a v i}^{\prime}=\frac{1}{p} \sum_{j=1}^{p} q_{i j}$ can be obtained.

In this way, we can determine the importance of the degree of relationships of $W_{c}$ from the information features. Take the information feature $c_{q i}$ as an example. The ANP method is employed to obtain the vector of the relative degree of importance of the information features. This is represented by $w_{i}=\left(w_{1 i}, w_{2 i}, \ldots, w_{i i}, \ldots\right.$, $\left.w_{p i}\right)^{T}$, in which $\sum_{j=1}^{p} w_{j i}=1$. We can use this to solve the relation matrix $\boldsymbol{W}_{c}$ to determine the degree of importance of the information features:

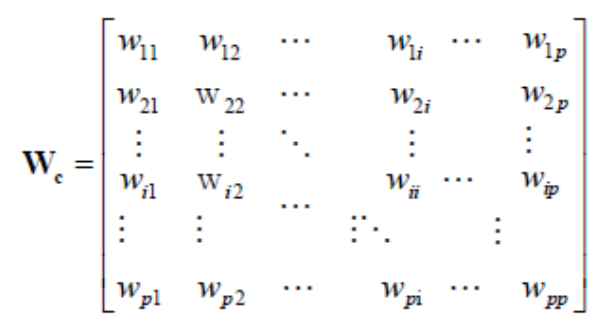


Finally, from this the degree of importance of the design information $w_{q}^{\prime}=W_{c} \times w_{c}=\left(w_{q 1}, w_{q 2}, \ldots, w_{q p}\right)^{T}$ can be determined. The eventual design information $C_{Q}=\left\{c_{q 1}, c_{q 2}, \ldots, c_{q n}\right\}$ and the corresponding vector for the degree of importance of the design information (after normalization) can be written as $w_{Q}=\left(w_{1}, w_{2}, \ldots\right.$, $\left.w_{n}\right)^{T}$.

\subsection{Mapping from Error-Cognition to the Design Information}

The relationship between the degrees of importance of the error-cognition and the design information is denoted by $W_{q e}$. When determining this relationship, we suppose that each design information is irrelevant, with respect to every item of the error factors. Then we compare the design information with each other, and the relationship matrix to obtain the relevant error factor. Then the Analytic Hierarchy Process (AHP) method is used to find solutions to the vector of the degree of importance for each design information for that error factor. This is represented as $w_{q e}=\left(w_{1 i}, w_{2 i}, \ldots, w_{i i}, \ldots, w_{p i}\right)^{T}$. Regarding the vector of the degree of importance for each design information of each error factor, the relation matrix $W_{q e}$ of the error-cognition and the design information is constructed as follow:

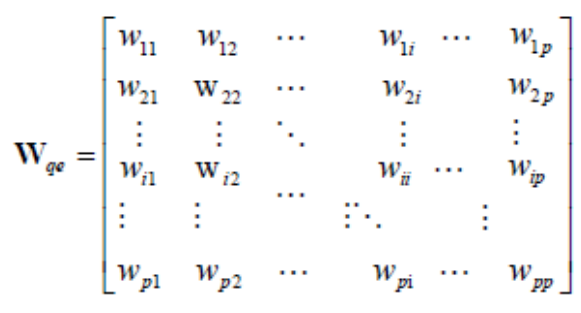

From the matrices $W_{q e}$ and $\mathrm{W}_{\mathrm{q}}$, we obtain the relationship matrix between the error-cognition and the design information, while considering the correlation among the design information as follows:

$$
W_{\mathrm{QE}}=W_{\mathrm{q}} \times\left(W_{\mathrm{qe}}\right)^{\mathrm{T}}
$$

Comprehensively, considering the influence of the degree of importance of error-cognition on the design information, the vector of the degree of importance of the design information is obtained as follow:

$$
W_{\mathrm{Q}}=W_{\mathrm{QE}} \times w_{\mathrm{E}}
$$

\subsection{Objective Constraints of Interface Layout}

When optimizing the contents of information presentation, in addition to considering the degrees of importance of the features of design information it is also necessary to consider the rationality of information features in the interface layout. The interaction interface includes all kinds of information fragments, such as symbols, indicators, line symbols, characters, etc, which are assembled to become an information set from the information flow. Ibrahim (2013) states information structure is the form of information assembled. So in this paper, from the point of information instruction, combined with Parsons' (2014) 10 attributes of interacting visual display, we can define information blocks, functional divisions, task divisions and visual flows as design information features of interface layout. According to the objective programming approach, we establish a mathematical model for the optimization and decision-making for the design information features in the interface layout (Hu Yunquan et al., 1998). We determine the objective constraints of design information features from several aspects as below:

1) Information capacity inside the interface area. The information units related graphics could be assembled as an information block. For example, different tactical models, such as Attacking, Defense, Expansive, etc. could be combined as one closely arranged information unit. In the monitoring task interface, this information block could be combined as unit A: $\{\mathrm{x} 1, \mathrm{x} 2, \ldots \ldots \mathrm{xn}\}$. There are Block A1, Block A2, Block A3, .... Block A , which could be formed into an Information (Info) Unit, that is Info: $\{A 1, A 2, \ldots \ldots$ An $\}$. According to the characteristics of information interaction systems, it is known that as the complication and size of 
information increases, so does the difficulty of the information layout. Hence, the quantity of information inside the interface, namely the information capacity, is a factor to be considered for design optimization. The amount of information can be quantified by a bit (Shannon and Weaver, 1949). With respect to the probability that the information occurs in the interface area, the capacity of different interface areas can be computed as follows:

$$
H_{\text {ave }}=\sum_{i=1}^{n} p_{i}\left[\log _{2}\left(1 / p_{i}\right)\right]
$$

To serve as a constraint, we set the probability of events that can possibly happen during the corresponding execution of a task for all kinds of information features. Then we calculate the average information quantity of these information features.

2) Visual flow of interface information: The visual behavior of operators forms a certain visual flow in the interface. This is the second factor that is important for design optimization. According to visual behavior rules (Wickens, 2003) and eye tracking, the visual flow mainly includes sensitivity of stimulus, guidance of physical location, edge effect and the gaze-saccade process.

3) The degree of relevance of information functions: With respect to the difference between functions, an internal graph-element relation has been formed among the information. This will form information blocks that are relatively concentrated in the interface. This is the third factor for design optimization. Based on information blocks, we need to consider the functional correlation among information blocks. For examples, the speed controlling area is comprised of airspeed, height, heading, etc. The weapons mounting selective area is comprised of class, selection marking, etc. The function division area could be designated unit B: $\{y 1, y 2, \ldots \ldots y n\}$. There are Area B1, Area B2, Area B3, ..... Area An, which could be formed function Area (Func) Unit, that is Func: $\{\mathrm{B} 1, \mathrm{~B} 2, \ldots \ldots \mathrm{B} n\}$.

4) The tasks are partitioned according to their degrees of importance: It is also necessary to consider the degrees of importance of the tasks in the interface layout. For different combinations of tasks, different task partitions are required. Hence task level is the most crucial factor in the interface layout. Based on the function division, we also need consider the operator's monitoring task. The monitoring task could be divided into different areas according to different processing of monitoring/detection, state query, response planning and response execution. There is a direct connection between the task division and the function division, such as fuel gage searching, indication radar setting and selection, weapon plan and bomb mode selection, etc. Therefore, the task division area could be as unit $\mathrm{C}:\{\mathrm{P} 1, \mathrm{P} 2, \ldots \ldots \mathrm{P} n\}$.

\subsection{Mathematical model and modeling procedures of objective programming}

Combining the aforementioned analysis, we identify four constraints: 1) information capacity, 2) visual flow, 3) graph-element relations and 4) task level. They are categorized into quantitative constraints (information capacity) and qualitative constraints (visual flow, graph-element relations and task level). The weighted multi-objective programming method is adopted to obtain the optimal information feature set for design optimization. The general mathematical model of objective programming can be written as follow:

$$
\min \omega_{1}\left(\mathrm{~d}_{1}^{-}\right)+\sum_{i=2}^{k} \omega_{1}\left(\frac{d_{i}^{-}}{R_{i}}+\frac{d_{i}^{+}}{R_{i}}\right)+\sum_{i=k+1}^{m} \omega_{i}\left(d_{i}^{-}\right)
$$

s.t. $\quad \sum_{j=1}^{n} w_{D j} x_{j}+d_{1}^{-}-d_{1}^{+}=1, \sum_{j=1}^{n} r_{i j} x_{j}+d_{i}^{-}-d_{i}^{+}=R_{i} \quad i=1,2, \ldots, s$, and $\sum_{j=1}^{n} w_{i j} x_{j}+d_{i}^{-}-d_{i}^{+}=1 \quad i=s+1, \ldots$,

$m$.

where, $\omega_{i}$ stands for the weight of an objective $(i=1,2, \ldots, m), d_{i}^{-}$represents the negative deviation to 
the $i$-th objective, $d_{i}^{-}$represents the positive deviation to the $i$-th objective, $x_{j}$ is the $0-1$ variable, that stands for the $j$-th feature of the design information $(j=1,2, \ldots, n), w_{D j}$ stands for the degree of importance for the $j$-th feature of the design information, $r_{i j}$ represents the $j$-th feature of the design information that uses the $i$-th quantitative constraint, $R_{i}$ stands for the $i$-th quantitative constraint, and $w_{i j}$ represents the $j$-th feature of the design information with respect to the weight of the $i$-th qualitative constraint objective.

\section{Case of Task Interface Design of Typical Avionics Display Systems}

\subsection{Error Factor and Extraction of Information Features of a Typical Avionics Display Interface}

In order to verify the reasonability of our design method for mapping from error-cognition to design information, we will take the surveillance task interface of a complex information system as an example. This paper takes information features of the surveillance task interface of an avionics display system for analysis (as depicted in Figure 2), in which the error factors are extracted based on the monitoring interface tasks and corresponding error factors (Table 1) as follows:

1) Visual restriction - omission $\left(r_{e 1}\right)$;

2) Visual mistake - misreading/misjudgment $\left(r_{e 2}\right)$;

3) Visual interference - ignorance $\left(r_{e 3}\right)$;

4) Attention shifting and distraction - miss $\left(r_{e 4}\right)$;

5) Cognitive deviation - misunderstanding $\left(r_{e s}\right)$;

6) Unreasonable matching - confusion $\left(r_{e 6}\right)$.

With respect to six items of error factors, the designers target the monitoring tasks executed by the operators, and combine them with features from design information to determine seven items from the design information features:

1) Location of an information feature $\left(c_{q 1}\right)$;

2) Visible range of an information feature $\left(c_{q 2}\right)$;

3) Spacing of information features $\left(c_{q 3}\right)$;

4) Intensity of visual attention of an information symbol $\left(c_{q 4}\right)$;

5) Recognition of an information icon $\left(c_{q 5}\right)$;

6) Degree of conciseness of an information icon $\left(c_{q 6}\right)$;

7) Differences between information icons $\left(c_{q 7}\right)$.

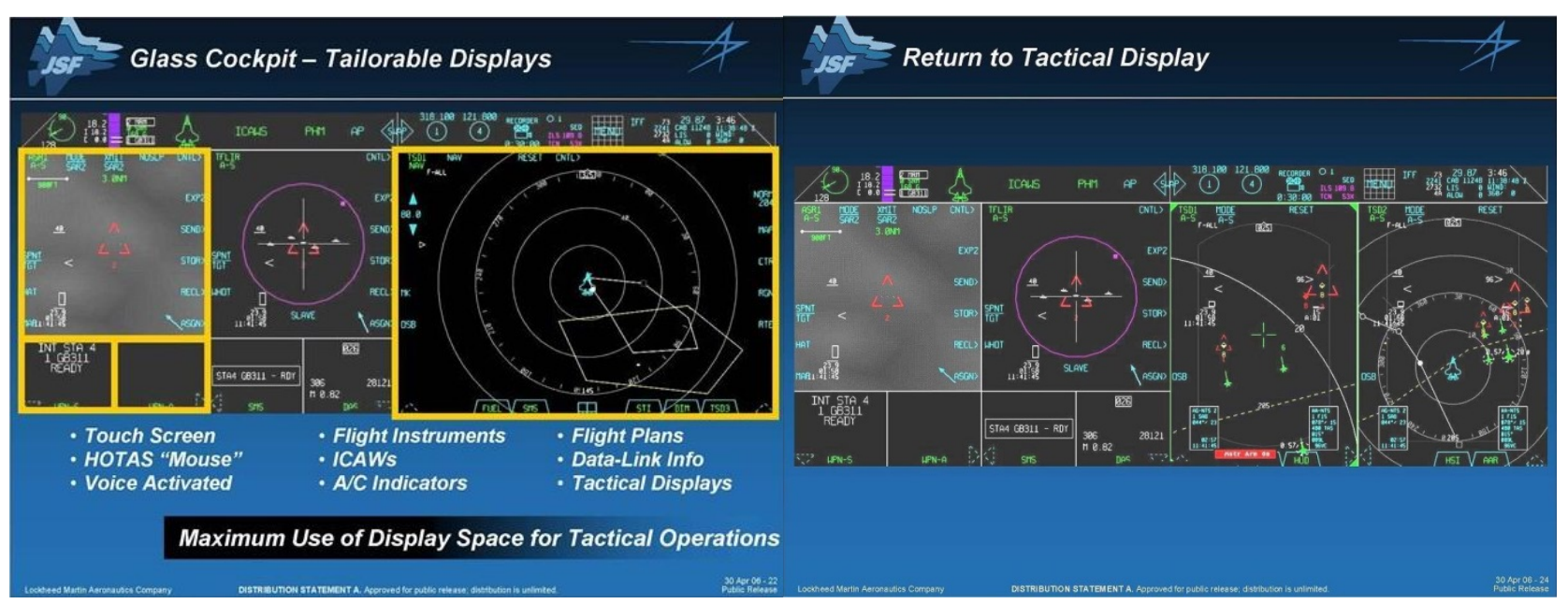

Figure 2. Monitoring Tasks: Mode 1 (left) and Mode 2 (right) 
Below, we will focus on six items of error factors from these typical error-cognition sets and seven items of design information features extracted by surveillance tasks executed by operators. The analysis will be conducted one by one with respect to the reaction chain. The optimizations of the intensity of visual attention are performed mainly with respect to the line symbol, the character symbol, and a combination of the line and character symbols. We also consider the significance, such as the allocation of colors and line frame symbols which affect the intensity of the visual attention. The relevant symbols of design information are illustrated in Figure 3.

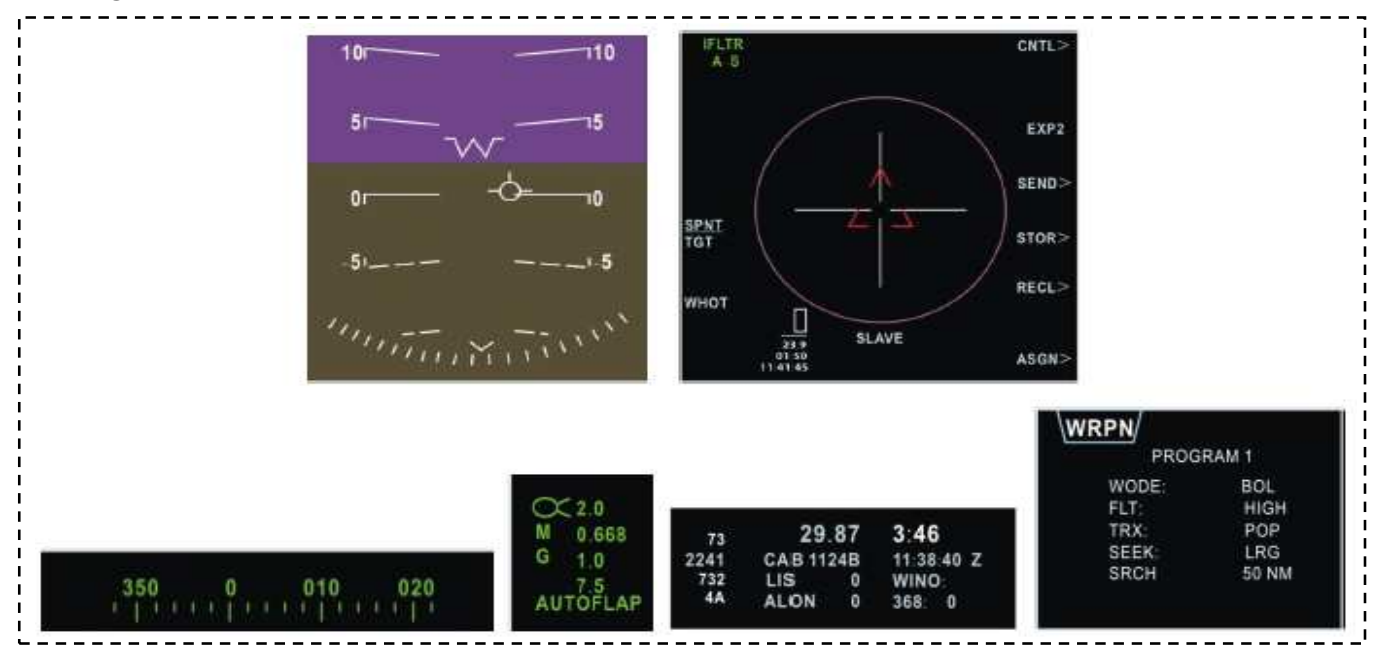

Figure 3. Information blocks with relatively weaker visual attention

The optimization of the recognition and the distinction of information graphic symbols are mainly from the points of understanding, degree of cognition, similarity of graphic symbols. The relevant symbols of design information are shown in Figures 4-a, 4-b, 4-c.

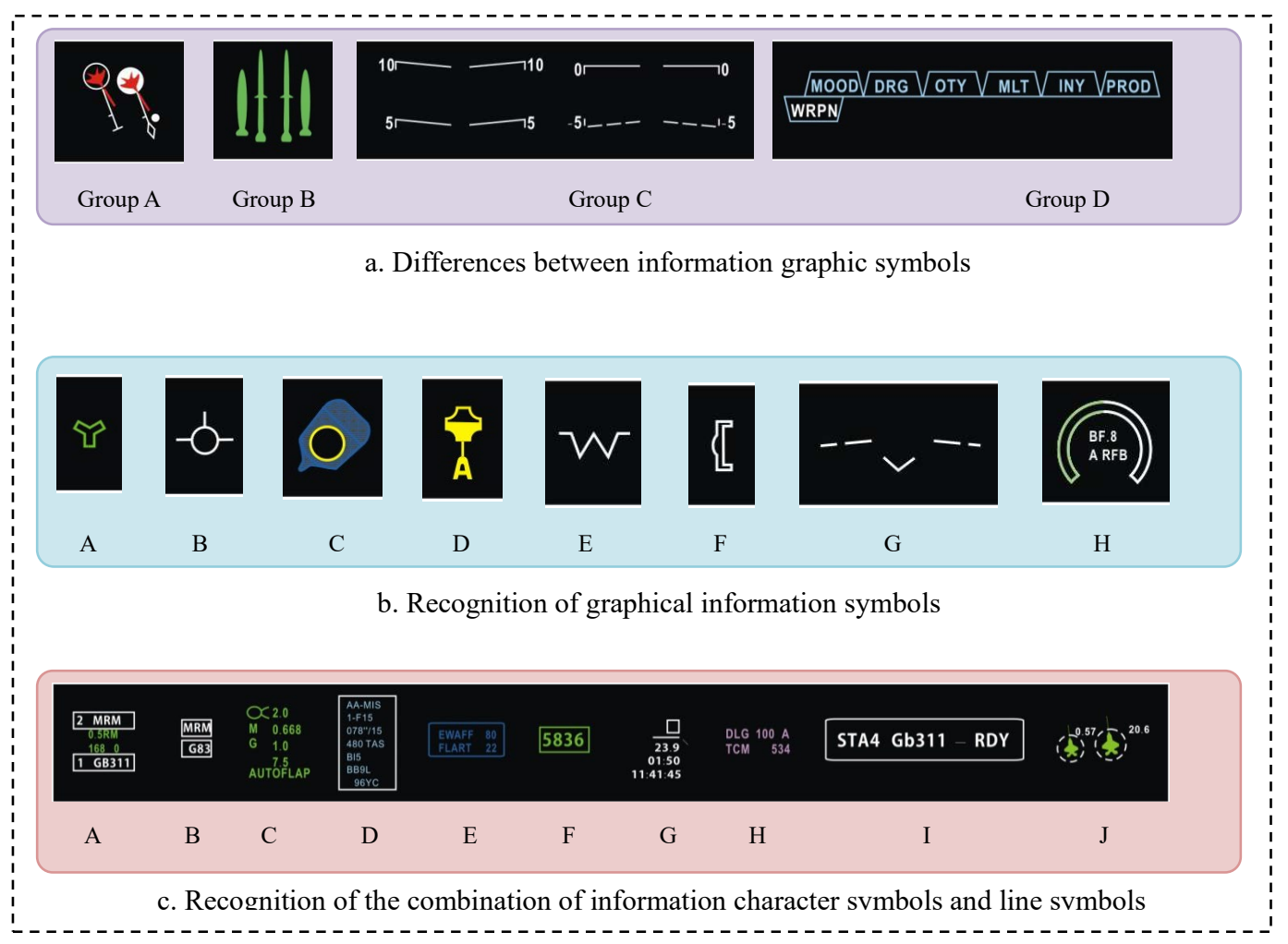

Figure 4. Information symbols 
With respect to the characterization methods of design factors for interface layout (Wu Xiaoli, 2015), the information structure of the interface can be extracted via abstract layouts. As a result, a layout analysis of the original interface results in the output of the layout abstracts of each sub-interface as depicted in Figure 4 (A, B, C and D).

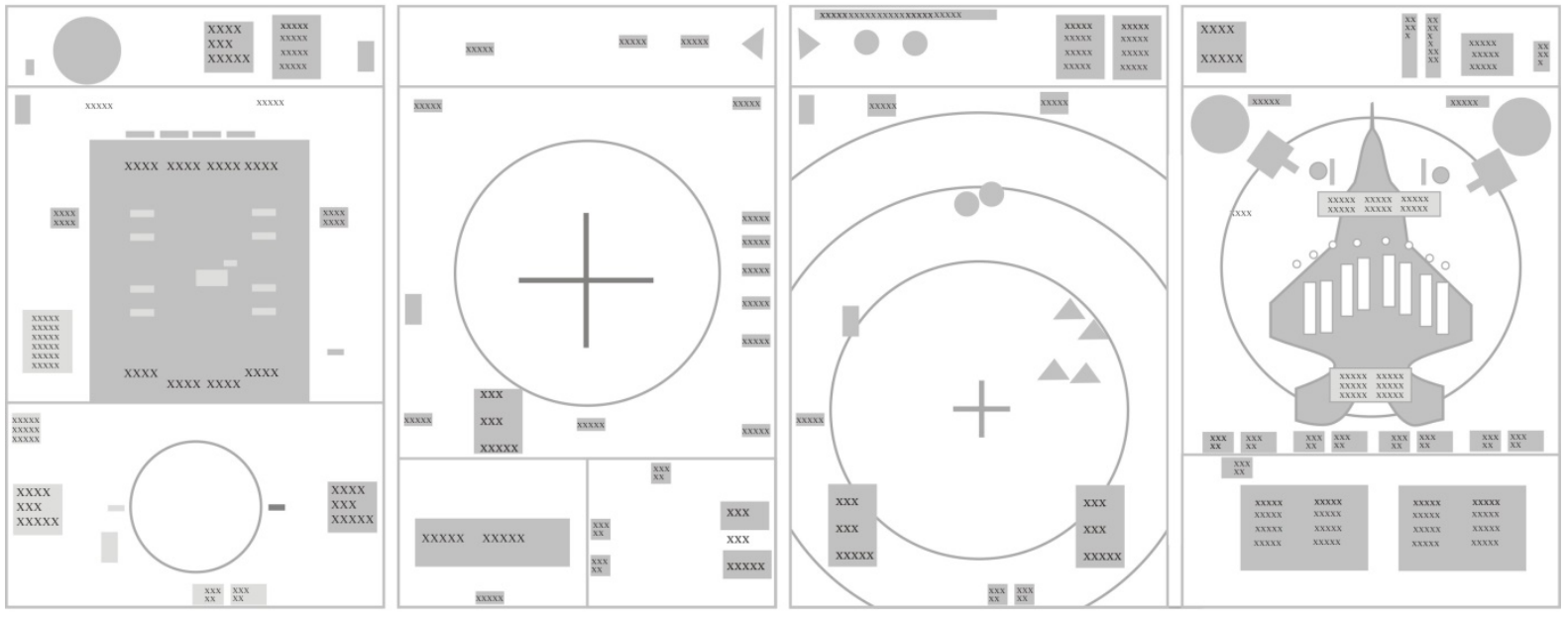

A. Display of flight data

B. Display of horizontal location

C. Radar status

D. Weapons mounting

Figure 5. The information layout of a monitoring task interface

It can be seen from the information structure of the interface that this layout can be partitioned into four visual districts, and these districts are divided equally in such a way that they lack concern for visual searching behaviors. What needs to be considered for the factors of information layouts are the locations of information features $\left(c_{q 1}\right)$, the visible ranges of information features $\left(c_{q 2}\right)$ and the intervals of the information features $\left(c_{q 3}\right)$.

\subsection{Mapping from error-cognition to design information}

With respect to the mapping method from error-cognition to the features of design information, the procedure to obtain the optimized design information is set as below:

Step 1: Determine the relative weights of the error factors for the error-cognition. Suppose the error factors denoted by the numerical sequence of 1 to 9 are irrelevant. Then compare five items of error factors to get the solution such that $\left(r_{\mathrm{e} 1}, r_{\mathrm{e} 2}, r_{\mathrm{e} 3}, r_{\mathrm{e} 4}, r_{\mathrm{e} 5}, r_{\mathrm{e} 6}\right)=(3,7,5,3,9,1)$. After normalization, we obtain the relative weighted vector of error factor $w_{r}$, where $w_{r}=[0.107,0.250,0.179,0.107,0.321,0.036]^{T}$.

Step 2: Determine $\mathbf{W} r$, the relationship matrix of the degrees of importance of the error factors:

$$
\mathbf{W} r=\left[\begin{array}{llllll}
0.652 & 0.000 & 0.148 & 0.183 & 0.016 & 0.000 \\
0.000 & 0.619 & 0.000 & 0.016 & 0.127 & 0.239 \\
0.148 & 0.000 & 0.503 & 0.309 & 0.023 & 0.019 \\
0.184 & 0.015 & 0.309 & 0.492 & 0.000 & 0.000 \\
0.016 & 0.127 & 0.023 & 0.000 & 0.577 & 0.255 \\
0.000 & 0.239 & 0.017 & 0.000 & 0.257 & 0.487
\end{array}\right]
$$

Step 3: Determine the relationship matrix of the degrees of importance between the error factors and the features of design information: 


$$
\mathbf{W} q e=\left[\begin{array}{lllllll}
0.538 & 0.327 & 0.106 & 0.029 & 0.000 & 0.000 & 0.000 \\
0.123 & 0.109 & 0.012 & 0.286 & 0.242 & 0.011 & 0.217 \\
0.107 & 0.077 & 0.118 & 0.497 & 0.000 & 0.000 & 0.201 \\
0.271 & 0.108 & 0.015 & 0.606 & 0.000 & 0.000 & 0.000 \\
0.000 & 0.000 & 0.000 & 0.000 & 0.579 & 0.138 & 0.283 \\
0.000 & 0.000 & 0.000 & 0.000 & 0.202 & 0.126 & 0.672
\end{array}\right]
$$

Step 4: Determine the relationship matrix of the degrees of importance of the features of design information:

$$
\mathbf{W} q=\left[\begin{array}{lllllll}
0.723 & 0.234 & 0.029 & 0.000 & 0.014 & 0.000 & 0.000 \\
0.234 & 0.527 & 0.135 & 0.104 & 0.000 & 0.000 & 0.000 \\
0.029 & 0.135 & 0.660 & 0.049 & 0.116 & 0.011 & 0.000 \\
0.000 & 0.104 & 0.049 & 0.548 & 0.012 & 0.129 & 0.158 \\
0.014 & 0.000 & 0.116 & 0.012 & 0.546 & 0.197 & 0.115 \\
0.000 & 0.000 & 0.011 & 0.129 & 0.197 & 0.506 & 0.157 \\
0.000 & 0.000 & 0.000 & 0.158 & 0.115 & 0.157 & 0.570
\end{array}\right]
$$

Step 5: Calculate $w_{E}$, the weight of an error factor:

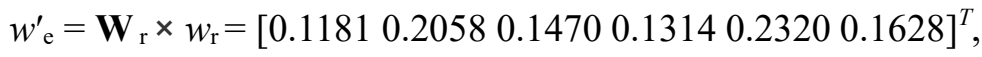

$$
\begin{aligned}
& R_{E}=\{3,7,5,3,9,1\} \text {, } \\
& w_{E}=[0.107,0.250,0.179,0.107,0.321,0.036]^{T} .
\end{aligned}
$$

Step 6: Calculate $w_{Q}$, the vector of the degrees of importance for the features of design information:

$$
\mathbf{W}_{Q E}=\mathbf{W}_{q} \times\left(\mathbf{W}_{q e}\right)^{T}=\left[\begin{array}{ccccccc}
0.4734 & 0.1193 & 0.0998 & 0.2241 & 0.0081 & 0.0028 \\
0.3155 & 0.1176 & 0.1332 & 0.1854 & 0.0000 & 0.0000 \\
0.1311 & 0.0684 & 0.1157 & 0.0620 & 0.0687 & 0.0248 \\
0.0551 & 0.2073 & 0.3179 & 0.3441 & 0.0695 & 0.1249 \\
0.0202 & 0.1658 & 0.0443 & 0.0128 & 0.3759 & 0.2124 \\
0.0049 & 0.1243 & 0.0970 & 0.0783 & 0.2283 & 0.2091 \\
0.0046 & 0.0868 & 0.0895 & 0.0951 & 0.1044 & 0.0813
\end{array}\right] .
$$

Step 7: Consider the quantitative objective constraints. For information features, information capacity is regarded as the quantitative constraint. Generally, in multi-dimensional comprehensive conditions, the transmission rate can be enhanced. However, they will always be below $10 \mathrm{bit} / \mathrm{s}$. This is the limit of the human information transmission rate - information transmitted above this rate cannot be fully comprehended. In a complex information interface, the transmission rate of information is influenced by multiple factors such as the size of graphical symbols, color, position and connecting line symbols. These factors are called stimulus dimensions (Ding Yulan, 2000). Thus, the information transmission rate which is optimal for operators to comprehend is not a constant. It changes with the features and the dimensions of the different graphical information symbols and the complexity of the task to be executed.

Hence, for different tasks to be executed by operators, the visual cognition processes from monitoring /discovery, status inquiry, response planning to response execution can take between 30 to 180s. This may sometimes lead to situations where it is possible to miss the optimal time for task execution. As a result, we can say that the information capacity to simultaneously perform information processing in an information interface should be within 300 to 1800 bits. If this capacity is exceeded, it becomes hard for operators to 
execute an information task. In this case, seven information features correspond to the task processes that need to be executed; they are $c_{\mathrm{q} 1}, c_{\mathrm{q} 2}, c_{\mathrm{q} 3}, c_{\mathrm{q} 4}, c_{\mathrm{q} 5}, c_{\mathrm{q} 6}$, and $c_{\mathrm{q} 7}$ respectively (see Table 3 ).

Table 3. Probabilities of occurrence $p_{i}$ of possible executed tasks corresponding to the information features

\begin{tabular}{ccccc}
\hline Information Features & A Monitoring/Discovery & B Status Inquiry & C Response Planning & D Response Execution \\
\hline cq1 & 0.55 & 0.25 & 0.125 & 0.175 \\
\hline cq2 & 0.35 & 0.45 & 0.08 & 0.12 \\
\hline cq3 & 0.25 & 0.55 & 0.175 & 0.025 \\
\hline cq4 & 0.35 & 0.35 & 0.125 & 0.175 \\
\hline cq5 & 0.25 & 0.25 & 0.375 & 0.175 \\
\hline cq7 & 0.125 & 0.225 & 0.35 & 0.3 \\
\hline
\end{tabular}

With respect to the computational formula for the average quantity of information

$H_{\text {ave }}=\sum_{i=1}^{n} p_{i}\left[\log _{2}\left(1 / p_{i}\right)\right]$, the computation can be performed as shown in Table 4 .

Table 4. Probabilities of occurrence of possible executed tasks corresponding to the information features

\begin{tabular}{ccccc}
\hline Information Features & A Monitoring/Discovery & B Status Inquiry & C Response planning & D Response Execution \\
\hline$p_{i}$ & 0.55 & 0.25 & 0.125 & 0.175 \\
\hline $1 / p_{i}$ & 1.82 & 4.00 & 8.00 & 5.71 \\
\hline $\log _{2}\left(1 / p_{i}\right)$ & 0.86 & 2.00 & 3.00 & 2.51 \\
\hline
\end{tabular}

The numerical value of $c_{q 1}$ is calculated as:

$$
\sum p_{i}\left[\log _{2}\left(1 / p_{i}\right)\right]=0.473+0.5+0.375+0.439=1.787 \text { bit. }
$$

Similarly, the size of the relevant information of $c_{q 2}, c_{q 3}, c_{q 4}, c_{q 5}, c_{q 6}, c_{q 7}$ can be calculated using the expression:

$\sum p_{i}\left[\log _{2}\left(1 / p_{i}\right)\right]$, as is shown:

$$
\begin{aligned}
& c_{q 1}=1.787 \\
& c_{q 2}=1.586 \\
& c_{q 3}=1.545 \\
& c_{q 4}=1.878 \\
& c_{q 5}=1.972 \\
& c_{q 6}=1.917 \\
& c_{q 7}=1.883
\end{aligned}
$$

The size of the information capacity as displayed in Table 3 does not take into consideration factors such as the quantity of information features of the same category. The quantity of different information features in the monitoring task interface is approximately between 1 and 100. For instance, the number of characters is between 20 and 80, and the number of graphical symbols is generally between 3 and 15 . Therefore, the information from each of the seven categories of information features is below 200 bits and the total information capacity of all the categories is between 300 and 1800 bits.

Taking the correlation between the information features into consideration, the capacity of information features $v$ can be expressed as follow: 


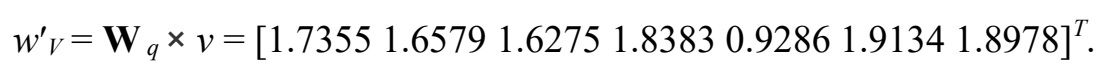

Step 8: Consider the qualitative objective constraints. We consider three objective constraints of information features. When carrying out interface layouts for design information these constraints are considered from the point of the visual flow, the graph-element relations and the task. For sufficient consideration of the optimization of design information with respect to the interface layouts, it is necessary to quantify the qualitative objectives. The method of "pairwise comparison" is used to compare seven items of information features. Next we obtain the weighted vector of each design information feature for the information capacity, visual flow, graph-element relations and task level considered in the interface layouts. They are denoted by $w_{\mathrm{F}}, w_{\mathrm{R}}$ and $w_{\mathrm{T}}$, respectively.

$$
\begin{aligned}
& w_{Q}=[0.327,0.195,0.155,0.254,0.037,0.011,0.021]^{T} \\
& w_{R}=[0.321,0.114,0.216,0.103,0.215,0.013,0.018]^{T} \\
& w_{T}=[0.328,0.187,0.073,0.373,0.011,0.011,0.017]^{T}
\end{aligned}
$$

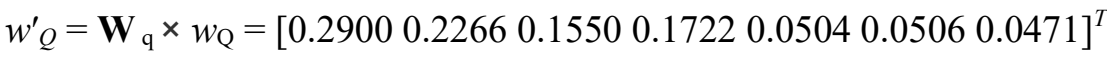

$$
\begin{aligned}
& w_{R}^{\prime}=\mathbf{W}_{\mathrm{q}} \times w_{\mathrm{R}}=\left[\begin{array}{lllllll}
0.2709 & 0.1751 & 0.1974 & 0.0860 & 0.1528 & 0.0674 & 0.0440
\end{array}\right]^{T}
\end{aligned}
$$

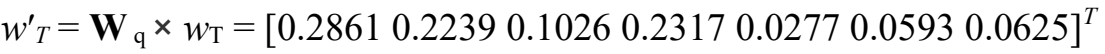

Step 9: Determine the relative weights of the objective constraints. Results calculated by "Pairwise comparison" are shown in Table 5, where "RMC" represents the objective mapping from error-cognition to the design information.

Table 5. Relative weight of various objective constraints

\begin{tabular}{c|c|c|c|c|c|c}
\hline & RMC & $\begin{array}{c}\text { Information } \\
\text { Capacity } \\
(v / \%)\end{array}$ & $\begin{array}{c}\text { Visual } \\
\text { flow } \\
(f / \%)\end{array}$ & $\begin{array}{c}\text { Graphic-Element } \\
\text { Relations }(r / \%)\end{array}$ & $\begin{array}{c}\text { Task Level } \\
(t / \%)\end{array}$ & $\begin{array}{c}\text { Relative } \\
\text { Weight } / \\
w_{\mathrm{r}}\end{array}$ \\
\hline RMC & 1 & 3 & 2 & $1 / 2$ & $1 / 3$ & 0.170 \\
\hline Information Capacity & $1 / 3$ & 1 & $1 / 2$ & $1 / 3$ & $1 / 5$ & 0.068 \\
\hline Visual Flow & $1 / 2$ & 2 & 1 & $1 / 2$ & $1 / 3$ & 0.118 \\
\hline Graphic-Element Relations & 2 & 3 & 2 & 1 & $1 / 2$ & 0.237 \\
\hline Task Level & 3 & 5 & 3 & 2 & 1 & 0.407 \\
\hline
\end{tabular}

Step 10: The weighted multi-objective programming model is established by using the results of the above-mentioned nine steps. The specific planning model is presented as below:

$$
\min : 0.17\left(d_{1}^{-}\right)+\frac{0.068}{1800}\left(d_{2}^{+}\right)+0.118\left(d_{3}^{+}\right)+0.237\left(d_{4}^{-}\right)+0.407\left(d_{5}^{-}\right)
$$

With respect to the solution process of multi-objective planning by Lingo, the solutions to $c_{q 1}, c_{q 2}, \ldots$, $c_{q 7}$ can be obtained, as is shown:

$$
\begin{aligned}
& c_{q 1}=0.2140 \\
& c_{q 2}=3.1820 \\
& c_{q 3}=0 \\
& c_{q 4}=0.6253 \\
& c_{q 5}=2.1667 \\
& c_{q 6}=0 \\
& c_{q 7}=0
\end{aligned}
$$

With respect to the results of multiple objective planning, four major information features, $c_{q 1}$ (the location of information feature), $c_{q 2}$ (the visible range of information feature), $c_{q 4}$ (the intensity of visual attention of information symbols feature), and $c_{q 5}$ (the recognition property of information graphic symbols feature) are regarded as the main objects of design optimization for this monitoring task interface. 


\subsection{Information display and interface design according to results of multi-objective planning}

According to visual behavioral characteristics (Ding Yulan, 2000) and visual searching model (Wickens and Holiands, 2003), vision is directed from left to right, from up to down, and from the upper left, the upper right, the lower left to the lower right. The distribution of interface layouts is shown in Figures 6, including the main task execution area (optimal visual zone), task execution reserve (secondary visual zone) and task execution reserve (third visual zone). The layout should maximize the task execution area, and hide non-execution areas. It could adjust different information block displays in respect of position and visual range.

\begin{tabular}{|c|c|c|}
\hline Navigation & Navigation & Navigation \\
\hline+ & ـ & \\
\hline Secondary Navigation & Secondary Navigation & Secondary Navigation \\
\hline \multirow[t]{2}{*}{$\begin{array}{l}\text { The Main Task Execution Area } \\
\text { (Optimal visual zone) }\end{array}$} & $\begin{array}{l}\text { Task Execution Reserve } \\
\text { (Secondary visual zone) }\end{array}$ & $\begin{array}{c}\text { Task Execution Reserve } \\
\text { (The third visual zone) }\end{array}$ \\
\hline & \multirow{2}{*}{$\begin{array}{c}\text { Related Information } \\
\text { of Auxiliary Task Reserve }\end{array}$} & \multirow{2}{*}{$\begin{array}{l}\text { Related Information } \\
\text { of Auxiliary Task Reserve }\end{array}$} \\
\hline Data Auxiliary View & & \\
\hline
\end{tabular}

Figure 6. The information optimized layout of the monitoring task interface

According to extraction of the error factors, analysis of visual behavior, the reaction chain of error factors and information features, and the results of multiple objectives planning, the avionics interface display can be optimized via the information symbols design and the information block layout. The 3 optimized modes interface display are shown in Figure 7 (Mode 1, Mode 2, and Mode 3 ).

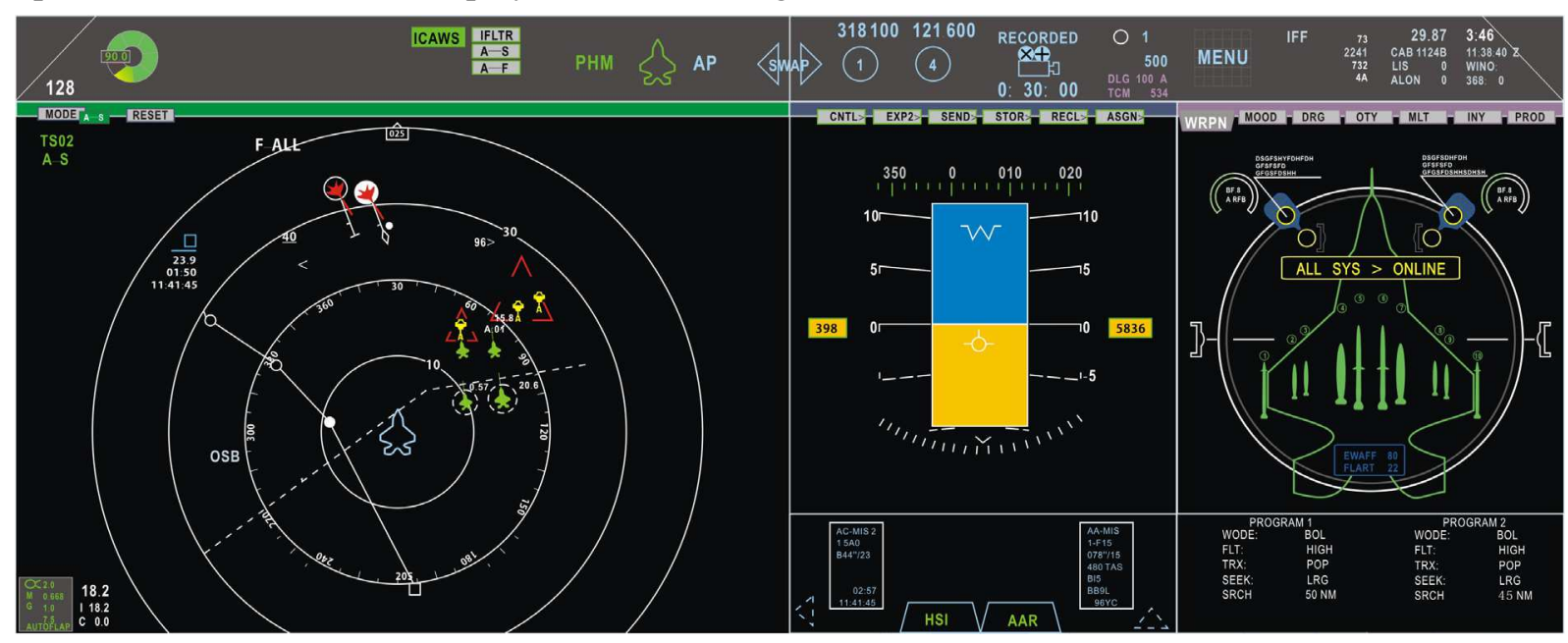

An optimized interfaces display (Mode 1) 


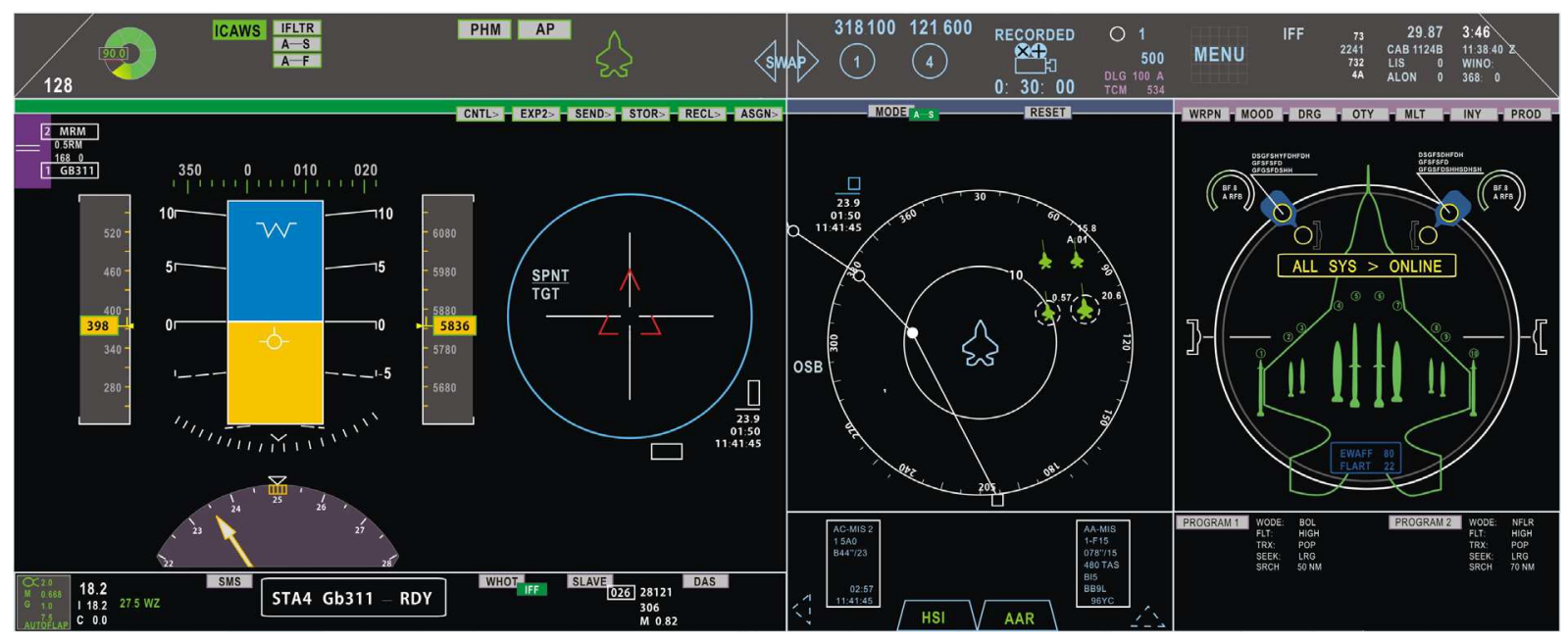

An optimized interface display (Mode 2)

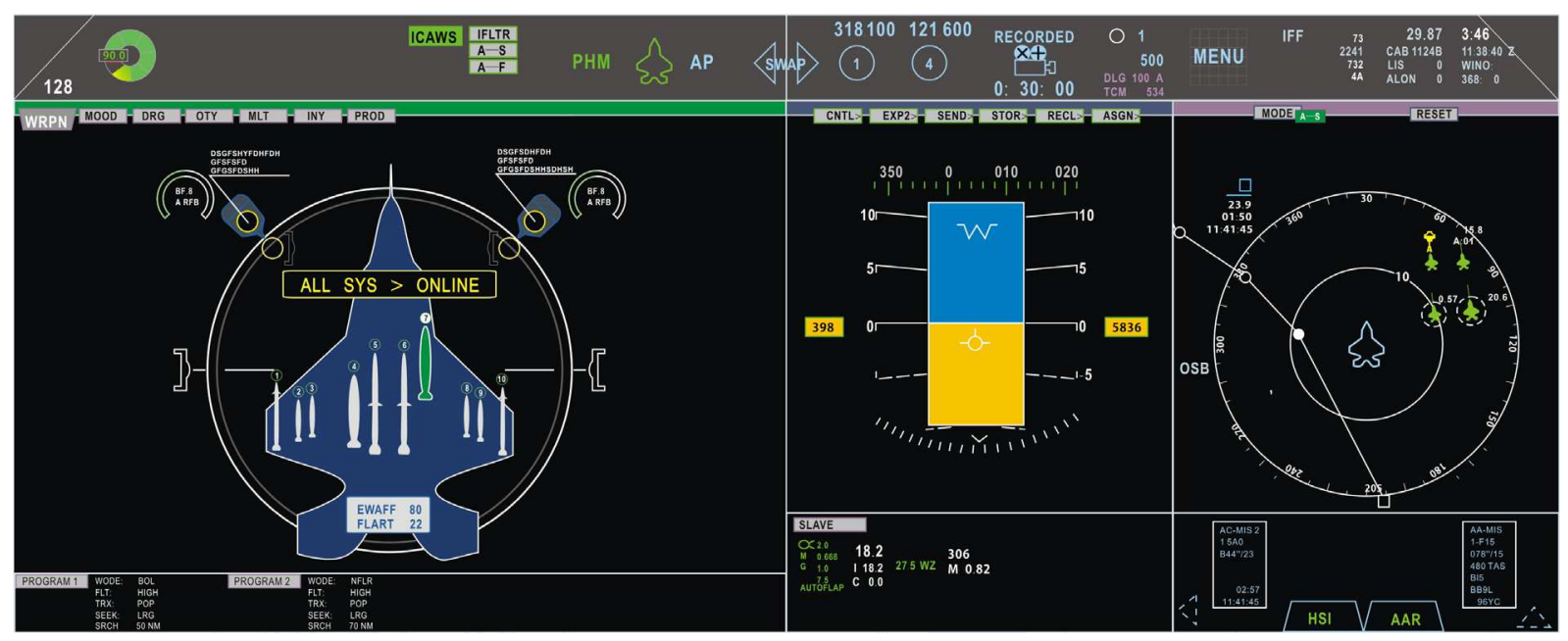

An optimized interface display (Mode 3)

Figure 7.3 optimized Modes interface display

\section{Conclusions}

This paper innovatively establishes the correlations among error factors, visual cognition and design information. It proposes that the interface design of complex information systems can directly benefit from the information derived from the sources of task failures. It also opens up a new shortcut towards the study of the design method of complex information interfaces, and builds up this design method from error-cognition to the mapping of information features.

With respect to the design method of complex information interfaces, we explore a use-case for the design of a typical naval warfare display system. Error factors such as omission, misreading/misjudgment, ignorance etc., are extracted along with the description of the relevant information features of tasks for the operators. Through the solution process of mapping, we identify four significant areas of information display design that can be improved in the monitoring task interface. These results verify the validity of the design method for information interfaces based on the mechanism of error-cognition.

The features of design information, such as locations of information characteristics, visual range, separation distance and visual attention intensity of symbols, are determined in accordance with the reaction 
chain of error factor-information characteristics. We find a solution to this problem by mapping from error-cognition to the information features. Based on the results, we also propose an optimization design scheme for the monitoring task interface.

\section{Key points}

Human computer interaction, Human Error, Visual Information Features, Error-cognition

\section{Acknowledgement}

This work was supported by Jiangsu Province Nature Science Foundation of China(BK20181159), the National Nature Science Foundation of China (Grant No. 71601068, 61603123), Science and technology projects of Changzhou (CE20175032), Jiangsu Province Key Project of philosophy and the social sciences

(2017ZDIXM023), Outstanding Young Scholars Joint Funds of Chinese Ergonomics Society- King Far (No. 2017-05), Overseas research project of Jiangsu Province (2017), and Fundamental Research Funds for the Central Universities (Grant No. 2015B22714)

\section{References}

Anokhin, A.N., and Marshall, E.C., 2009. The practice of main control room ergonomics assessment and validation using simulation tools. Proceeding of 6th American Nuclear Society International Topical Meeting on Nuclear Plant Instrumentation, Control, and Human-Machine Interface Technologies. Knoxville: American Nuclear Society, 2472-2483

BaoShan, Liu., 1987. Research on Simulating the Visual Effects of Various Visual Districts of Main Instrumental Board of Fighters. Acta Psychological Sinica, (1), 86-91.

Christopher, D,Wickens. and Justin, G, Holiands., 2003. Engineering Psychology \& Human Performance. East China Normal University Press.

Gero. J.S., 1996. Creativity, emergence and evolution in design. Knowledge-Based System, 9(7), 435-448.

Hassnert, M., and Allwood, C.M., 2002. Development context and ease of use of three programs for self-registration of unemployed people. Computers in human behavior, 18, 191-221.

Heather, F, Neyedli., Justin, G, Hollands., and Greg, A, Jamieson., 2011. Beyond identity: Incorporating system reliability information into an automated combat indentification system. Human factors: the journal of the human factors and ergonoimics society, 53(4), 338-355.

Ibrahim, Demir., Witold, F, Krajewski., 2013. Towards an integrated Flood Information System: Centralized data access, analysis, and visualization. Environmental Modelling \& Software, 50, 77-84.

Javier, Roca., Juan, Lupianez., and Maria-Fernanda, Lopez-Ramon., 2013. Are drivers' attentional lapses associated with the functioning of the neurocognitive attentional networks and with cognitive failure in everyday life? Transportation research part $F, 17,98-113$.

Jing, Li., Chengqi, Xue., Minghao Shi., Yafeng, Niu., and Haiyan, Wang., 2012. Information visual structure based on multidimensional attributes of information. Journal of southeast University ( Natural Science Edition), 42(6), 1094-1099.

Jing, Li., Chengqi, Xue., Haiyan, Wang., Lei, Zhou., and Yafeng, Niu., 2013. Encoding Information of Human-Computer Interface for Equilibrium of Time Pressure. Journal of Computer-Aided Design \& Computer Graphics, 2013,25(7): 1022-1028

Krokos, K.J., and Baker, D.P., 2007. Preface to the special section on classifying and understanding human error. Human factors, 49(2), 175-176.

Lee, J.W., and Kim, S.H., 2000. Using analytic network process and goal programming for interdependent information system project selection. Computer \&operation research, 27, 367-382.

Lei, Zhou., and Chengqi, Xue., 2013. Research of Interface Composition Design Optimization Based on Visual Balance. The 2013 International Conference on Intelligent Systems and Knowledge Engineering. Shenzhen, November 20-23, 279:483-493

Lei, Zhou., Chengqi, Xue., Wencheng, Tang., Jing, Li., and Yafeng, Niu., 2013. Aesthetic Evaluation Method of Interface Elements Layout Design. Journal of Computer-Aided Design \& Computer Graphics, 25(5), 758-766.

Leshan, Li., 2004. Design of Human-Computer Interfaces. Beijing: Science Press.

Liangming, Li., 1984. Investigation on Engineering Psychology of Aircraft Cockpit Instrument on the Flight Eight. Aviation Military Surgeon, (1), 60-61. 
Maxion, R.A., and Reeder, R.W., 2005. Improving user-interface dependability through mitigation of human error. International Journal of human-computer studies, 63(1-2), 25-50.

Men, Wang., Yijing, Zhang., Hua, Deng., Yunhua, Guo., and Bin, Wu., 2014. The Design of Software for Capturing Error During Manual Controlled Rendezvous and Docking Based on Cognitive Task Analysis. Industrial Engineering and Management. 19(5), 111-116

MIL-STD-1787B (USAF)., 1996. Aircraft Display Symbology. The Department of Defense of United States of America.

Michelle,Yeh., and Christopher, D, Wickens., 2001. Attentional Filtering in the Design of Electronic Map Displays: A Comparison of Color Coding, Intensity Coding, and Decluttering Techniques. Human Factors, 43 (4), 543-562.

Montgomery, D.A. and Sorkin, K.D., 1996. Observer sensitivity to element reliability in a multi-element visual display. Human Factors, 38(3), 484-494.

Nielsen, J., and Mack, R.L., 1994. Usability Inspection Methods. New York: Wiley.

Pahl, G., and W. Beitz., 1996. Engineering Design: A Systematic Approach. London: Springer-Verlag.

Parsons, P., and Sedig, K., 2014. Adjustable Properties of Visual Representations: Improving the Quality of Human-Information Interaction. Journal of the Association for Information Science and Technology, 65(3), 455-482

Patrick, Monnier., 2003. Redundant coding assessed in a visual search task. Displays, 24(1), 49-55.

Pei, Wang., and Pei-luen, Patrick, Rau., 2011. A Driver' s Perception and Comprehension of Traffic Signs in Beijing. Industrial engineering journal, 14(1), 114-117

Pengcheng, Li., 2011. Study on Human Error and Reliability in Digital Control System of Nuclear Power Plant. Changsha: Doctoral Dissertation of South China University of Technology.

Schum, D.A., 1991. The weighting of testimony in judicial proceeding from sources having reduced credibility. Human Factors, 33(2): 172-182.

Shannon, C.E., and Weaver, W., 1949. The mathematical theory of communications. Urbana: University of Illinois press.

Shappell, S., Detwiler, C., and Holcomb, K., 2007. Human Error and Commercial Aviation Accidents: An Analysis Using the Human Factors Analysis and Classification System. Human Factors, 49(2), 227-242

Shryane, N.M., Westerman, S.J., Crawshaw, C.M., Hockey, G.R.J., and Sauer, J., 1998. Task analysis for the investigation of human error in safety critical software design: a convergent methods approach. Ergonomics, 41(11), 1719-1736.

Suh, N.P., 2000. Axiomatic design advances and applications. New York: Oxford University Press.

Tao, Jin., and Chengqi, Xue., 2013. A new method of building an evaluation model for User Interface. $3^{\text {rd }}$ Interational Conference on Machinery, Materials Science and Engineering Applications, Wuhan, China, June, 20-12, 744, 605-609

Tao, Jin., Cengqi, Xue., Haiyan, Wang., and Yafeng, Niu., 2014. Research on Evaluating methods of Situation Awareness of Digital Graphical Interfaces. Chinese Journal of Engineering Design, 21(1), 87-91.

Tullis, T.S., 1981. An evaluation of alphanumeric, graphic, and color in-formation displays. Human Factors, 23(5), 541-550.

Xiaoli, Wu., 2017. Error-Cognition Mechanism of Task Interface in Complex Information System. Beijing: Science Press.

Xiaoli, Wu., 2015. Research on the Mechanism of Error-Cognition for Interaction Interface of Complex Information. Nanjing: Doctoral Dissertation of Southeast University.

Xiaoli, Wu., Chengqi, Xue., and Feng Zhou., 2014. Misperception Model-based Analytic Method of Visual Interface Design Factors. Engineering Psychology and Cognitive Ergonomics - 11th International Conference, EPCE 2014, Held as Part of HCI International 2014, Proceedings, Crete: 8532 LNAI, 284-292.

Xiaoli, Wu., Chengqi Xue, and Haiyan, Wang., 2014. E-C Mapping Model of Human-Computer Interaction Interfaces of Complicated Systems. Journal of Mechanical Engineering, 50(12), 206-212.

Xiaoli, Wu., Chengqi Xue, and Wencheng, Tang., 2014. Experimental study on visual limitation experiment of goal-seeking in radar situation-interface. Journal of southeast University ( Natural Science Edition), 44(6), 1166-1170.

Xiaoli, Wu., Yan, Chen., and Feng, Zhou., 2016. An Interface Analysis Method of Complex Information System by Introducing Error Factors. 13th International Conference on Engineering Psychology and Cognitive Ergonomics, EPCE 2016 and Held as Part of 18th International Conference on Human-Computer Interaction, HCI International, Toronto, Canada, July 17-22, 9736 LNAI, 116-124.

Xiaoli, Wu., Xue Chengqi., and Tang Wencheng., 2014. Study on eye movement of information omission misjudgment in radar situation-interface. Engineering Psychology and Cognitive Ergonomics - 11th International Conference, EPCE 2014, Held as Part of HCI International 2014, Proceedings, Crete: 8532 LNAI, 407-418

Yafeng, Niu., and Chengqi, Xue., 2014. Icon memory research under different time pressures and icon quantities based on event-related potential. Journal of Southeast University(English Edition), 30(1), 45-50.

Yaping, Ding., Baoshan, Liu., and Buping, Cao., 1994. Simulating the Visual Effects of Various Visual Districts of Main Instrumental Board of Military Helicopters. Journal on Aviation Medicine of China, 5(2), 108-111.

YunQuan, Hu., and Yaohuang, Guo., 1998. Tutorials on Operational Research. Beijing: Publication Press of Tsinghua 
University.

Yulan, Ding., 2004. Ergonomics. Beijing: Institute of Technology Press.

Zupei, Shen., Yao, Wang., and Jia, Gao., 2009. Theory and Manager Application of Human Error Cognition Model. Bei Jing: Tsinghua University. 
Figures

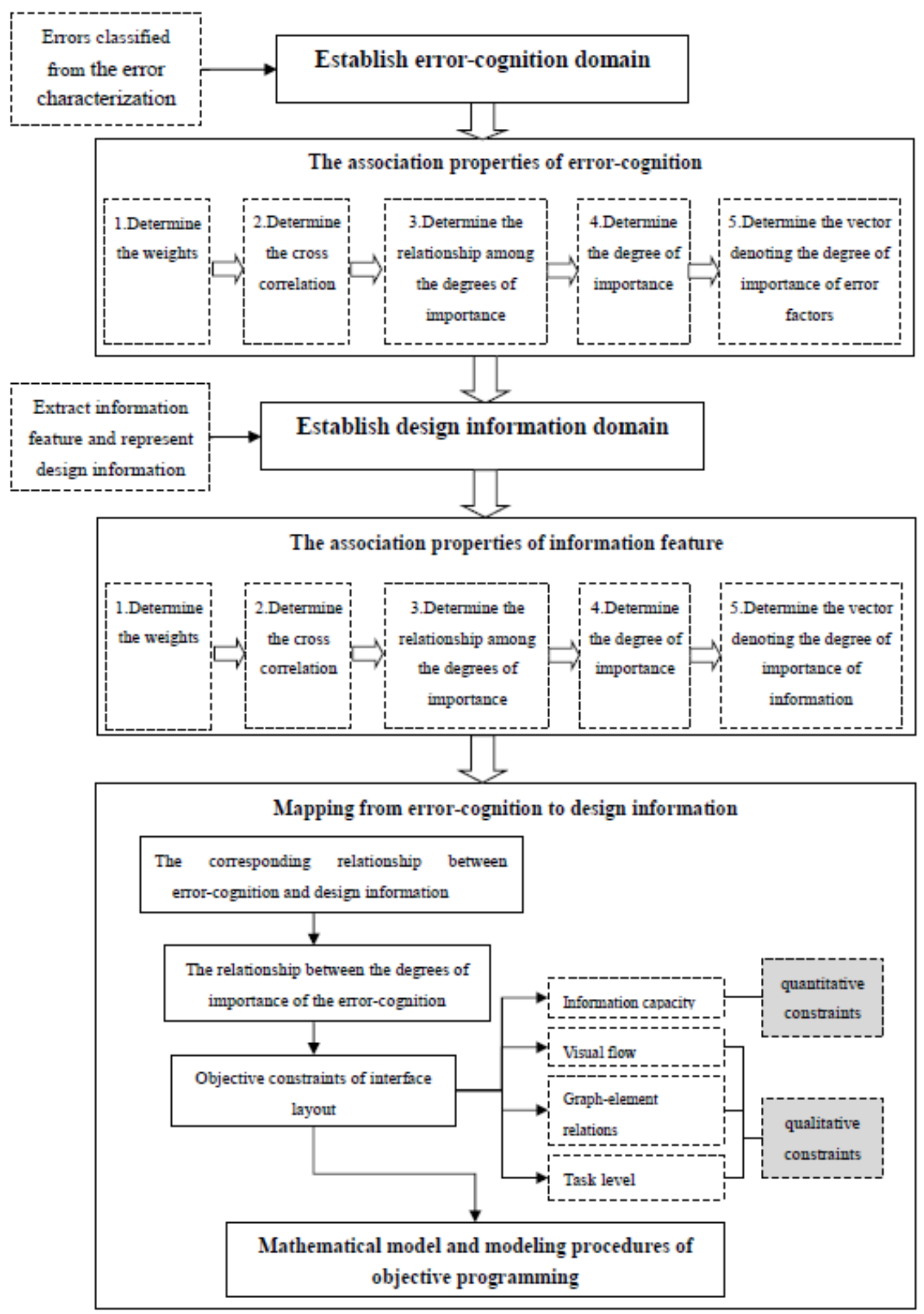

Figure 1

Procedure of the optimization method 

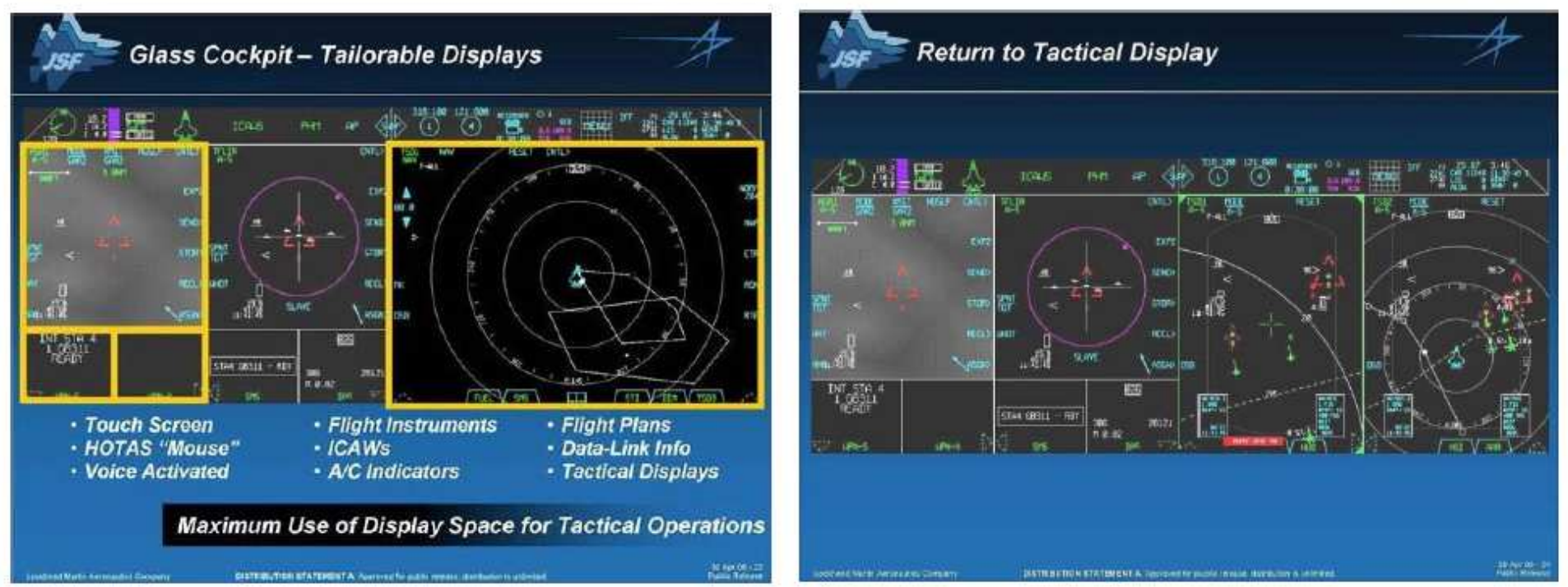

\section{Figure 2}

Monitoring Tasks: Mode 1 (left) and Mode 2 (right)

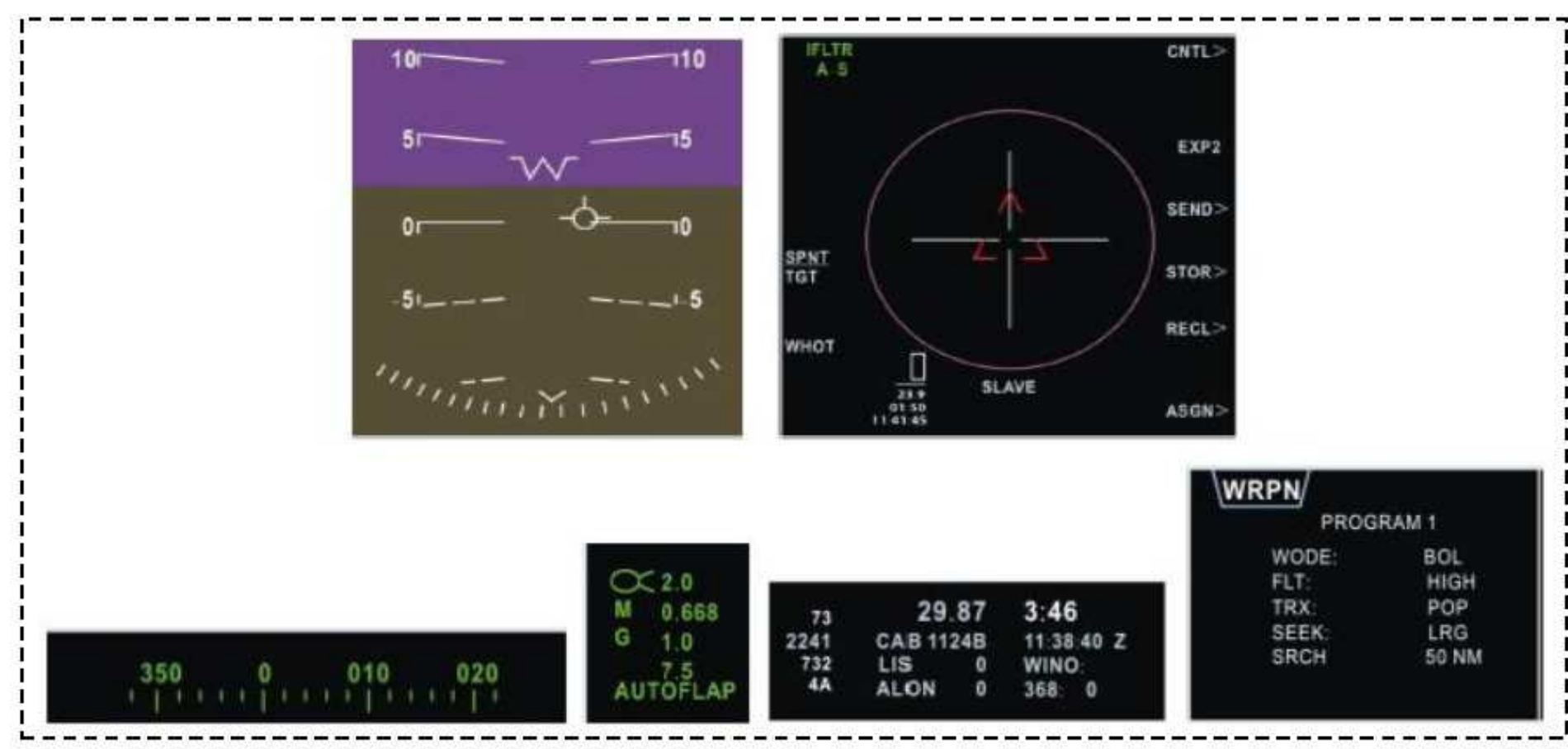

\section{Figure 3}

Information blocks with relatively weaker visual attention 


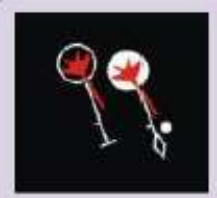

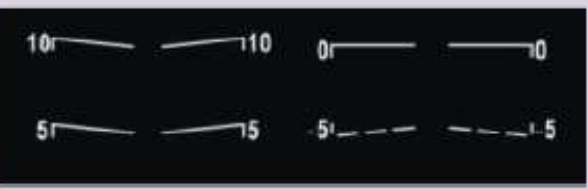

Group C
MOOD $\sqrt{\text { DRG }} \sqrt{\text { OTY }} \sqrt{\text { MLT }} \sqrt{\text { INY }} \sqrt{\text { PROD }}$ WRPN

Group A Group B

a. Differences between information graphic symbols

Group D
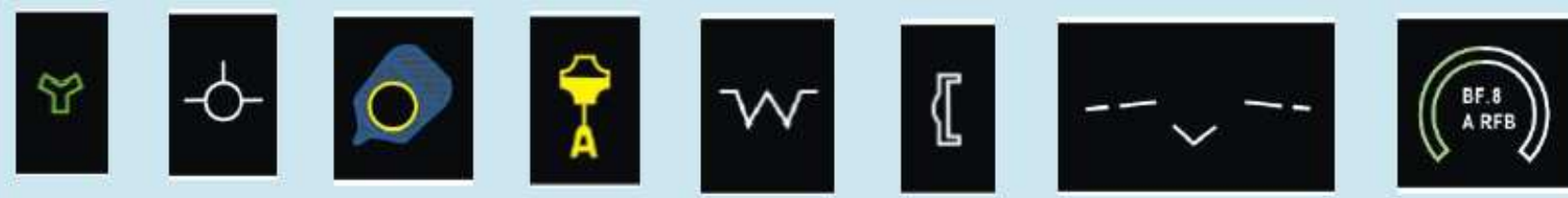

A

B

C

D

E

F

G

$\mathrm{H}$

b. Recognition of graphical information symbols

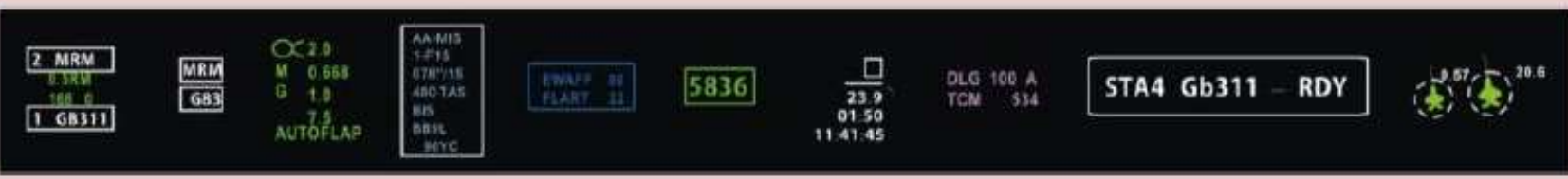

A

B

C

D

E

F

G

$\mathrm{H}$

I

J

c. Recognition of the combination of information character symbols and line svmbols

\section{Figure 4}

Information symbols
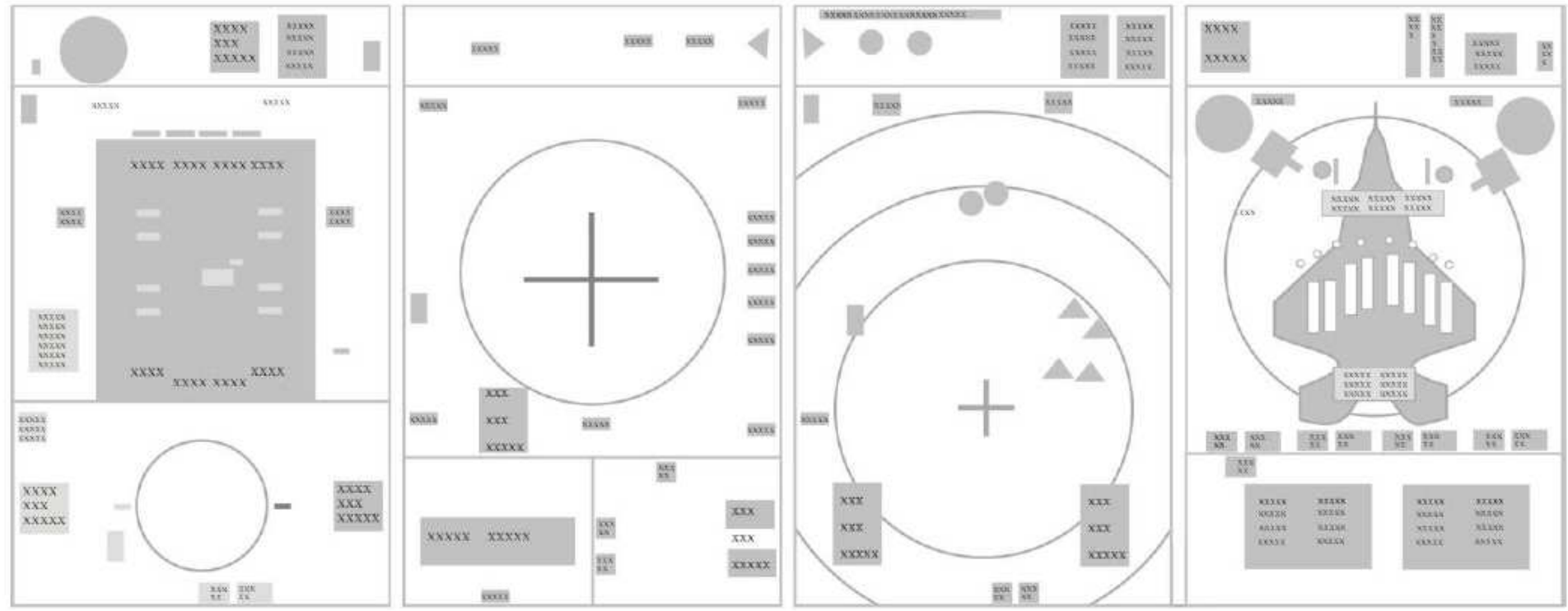
Figure 5

The information layout of a monitoring task interface

\begin{tabular}{|c|c|c|}
\hline Navigation & Navigation & Navigation \\
\hline - & $\sim$ & \\
\hline Secondary Navigation & Secondary Navigation & Secondary Navigation \\
\hline \multirow[t]{2}{*}{$\begin{array}{l}\text { The Main Task Execution Area } \\
\text { (Optimal visual zone) }\end{array}$} & $\begin{array}{c}\text { Task Execution Reserve } \\
\text { (secondary visual zone) }\end{array}$ & $\begin{array}{c}\text { Task Execution Reserve } \\
\text { (The third visual zone) }\end{array}$ \\
\hline & \multirow{2}{*}{$\begin{array}{l}\text { Related Information } \\
\text { of Auxiliary Task Reserve }\end{array}$} & \multirow{2}{*}{$\begin{array}{l}\text { Related Information } \\
\text { of Auxiliary Task Reserve }\end{array}$} \\
\hline Data Auxiliary View & & \\
\hline
\end{tabular}

\section{Figure 6}

The information optimized layout of the monitoring task interface 


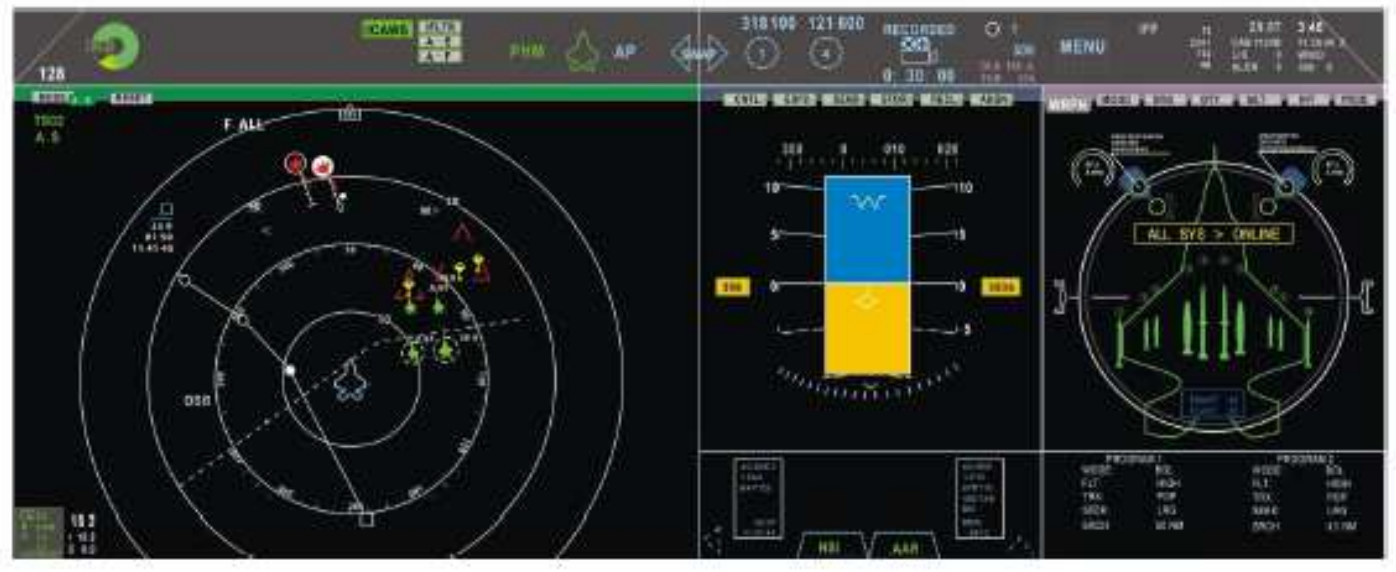

An optimized interfaces display (Mode 1)

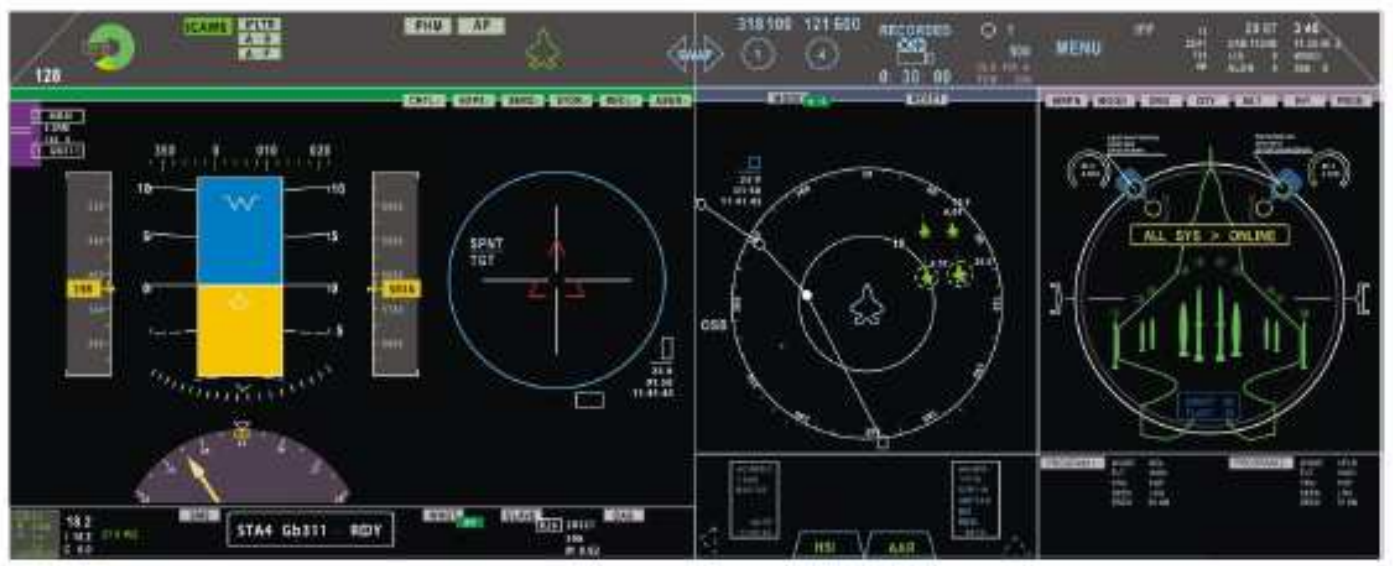

An optimized interface display (Mode 2)

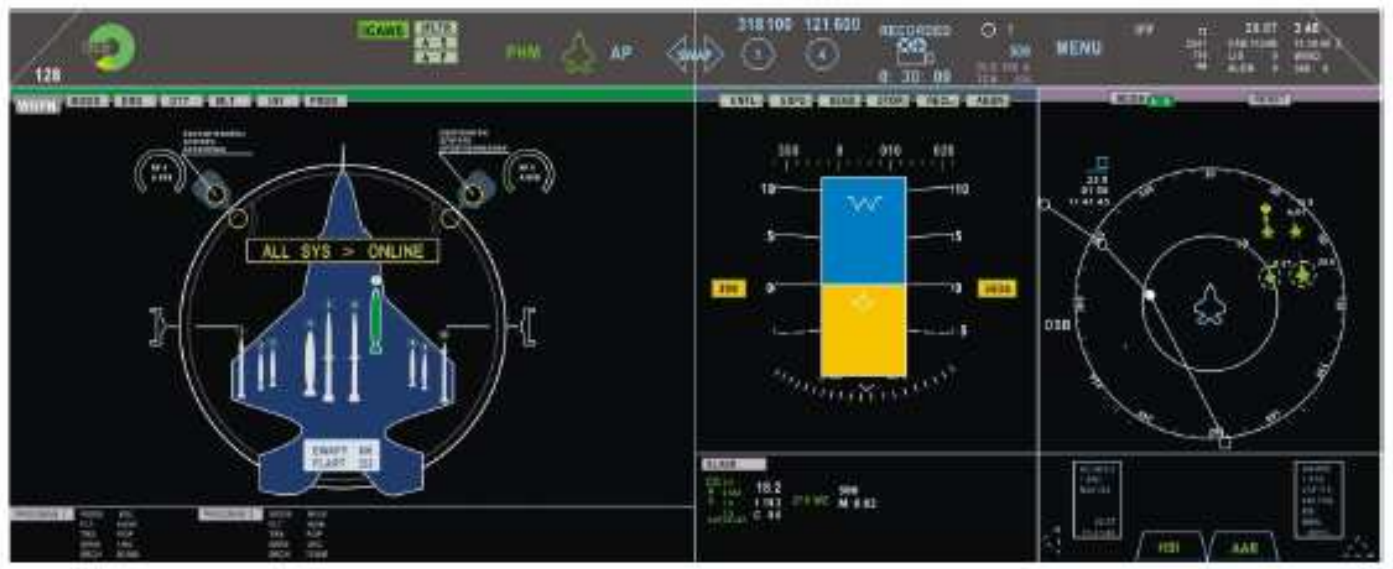

An optimized interface display (Mode 3)

\section{Figure 7}

3 optimized Modes interface display

\section{Supplementary Files}

This is a list of supplementary files associated with this preprint. Click to download. 
- supplementmaterial.doc 